\title{
Uniqueness and Existence of Equilibrium in Auctions with a Reserve Price
}

\author{
Alessandro Lizzeri* \\ Princeton University, Fisher Hall, Department of Economics, Princeton, \\ New Jersey 08544 \\ and \\ Nicola Persico ${ }^{\dagger}$ \\ Department of Economics, University of Pennsylvania, 3718 Locust Walk, \\ Philadelphia, Pennsylvania 19104-6297
}

Received June 12, 1997

We prove existence and uniqueness of equilibrium for a general class of twoplayer bidding games. We apply our results to the first price auction, the combination of first and second price auctions, the war of attrition, the all pay auction, as well as combinations of the latter two auction forms. We also treat the first price auction without risk neutrality. Our results deal with the asymmetric, affiliated common values environment. In the case where signals are independent our results apply to all equilibria. When signals are not independent, our uniqueness results hold in the class of nondecreasing strategy equilibria. Journal of Economic Literature Classification Number: D44. @ 2000 Academic Press

\section{INTRODUCTION}

We study a general model of bidding games. This accommodates most of the auction forms studied in the literature, more exotic ones such as the winner paying a combination of his and the loser's bid, and games of timing like the war-of-attrition. Our setup can also be used in

*E-mail: lizzeri@princeton.edu.

† E-mail: persico@econ.sas.upenn.edu.

We are grateful to two anonymous referees for useful comments. 
some examples of auctions with externalities $a^{\prime}$ la Jehiel and Moldovanu (1997).

This paper makes three contributions. The first is to address the question of uniqueness of equilibrium in auctions with interdependent values and a reserve price: this is important because reserve prices are commonly used in auctions, and it is well known that revenue can be increased by an appropriate choice of a reserve price.

The interdependence of valuations complicates the question of uniqueness of equilibrium in auctions (even without a reserve price), because the expected value of the object to bidder 1 conditional on winning depends on how aggressive bidder 2 is in his bidding. If bidder 2 is very aggressive, the winner's curse is particularly strong because bidder 1 wins only if bidder 2's signal is quite low. This makes bidder 1 optimally respond by being cautious in his bidding. Conversely, if bidder 2 is very cautious in his bidding, bidder 1 responds by being aggressive. Milgrom (1981) shows that this phenomenon generates a continuum of equilibria in undominated strategies in the second price auction, in sharp contrast with the dominance solvability of the second price auction in a world with private values. Common values introduce a similar problem in the treatment of reserve prices. Equilibrium bidding strategies are described by a system of differential equations representing the first-order conditions, coupled with initial conditions representing the types of players who bid at the reserve price. In auctions with private values, these initial conditions are uniquely determined by an indifference condition between entering and not entering the auction, and reserve prices add no serious complications to the analysis. In contrast, common value elements introduce a multiplicity of admissible initial conditions, which under certain conditions can be ranked by relative aggressiveness of the entry decision-a type of bidder 1 will find it more appealing to enter when a large fraction of types of player 2 stay out, since this increases type 1's payoff of winning against an opponent who stays out. This gives rise to a multiplicity of admissible initial conditions and therefore conceivably many equilibria. Indeed, a similar intuition underlies a multiplicity result for the war of attrition [Nalebuff and Riley (1985)].

We show that for a wide class of auction games this multiplicity of initial conditions does not translate into a multiplicity of equilibria. This is because in most auctions, at equilibrium the highest types of both players must bid the same finite amount (otherwise one of them would gain by reducing his bid). This "final condition" is precisely the property that fails in the war of attrition, where bidding is unbounded, but for all auctions where bidding is bounded we prove that this property guarantees uniqueness of equilibrium. The lesson we draw from this is that bidding games that are "uniqueness challenged," like the war of attrition or the second price auction, are really knife-edge cases in a large class of mechanisms. A small perturbation in 
the mechanism suffices to restore uniqueness. Our uniqueness results apply to all equilibria in the case where signals are independent (but still an element of common value exists). When signals are not independent, we prove uniqueness in the class of nondecreasing strategy equilibria.

While the "final condition" is helpful in the proof of uniqueness of equilibrium, it is an additional constraint to meet in proving existence of equilibrium. Our proof of existence of equilibrium is the second contribution of the paper. In the literature on the existence of equilibrium in discontinuous games [e.g., Dasgupta and Maskin (1986); Reny (1997)] auctions have proved hard to treat in a general and direct fashion. Our technique is constructive and follows easily from the reasoning that proves uniqueness.

The third contribution has a more technical nature. This paper presents a unified approach to characterizing the equilibrium set of a large class of bidding games. The ideas behind this approach are quite natural, and formalize considerations of "relative toughness" that are fundamental to strategic reasoning in all auction games. Due to this generality, we are able to understand exactly which features are necessary to obtain certain desirable properties of equilibrium strategies, such as continuity or differentiability. In this connection, it is worth remarking that the regularity analysis developed in the Appendix applies to all common auction forms, and is not limited to the model with reserve prices.

We treat the asymmetric case, which is of interest because it has been used to model bidding rings in an $n$-bidder symmetric auction with collusion, or to capture informational or technological differences among bidders. A limitation of our analysis is the restriction to two players: while this assumption is appropriate for some of the games we treat, like the war of attrition, or for defense procurement auctions where two competing consortia are frequent, further research is necessary in the $n$-bidders model.

\subsection{Related Literature}

Milgrom and Weber (1982) introduced the affiliation concept in auctions and studied symmetric first price, second price and English auctions. Milgrom and Weber discuss reserve prices but do not characterize the equilibrium set of the auctions they consider: they focus on symmetric equilibria. Krishna and Morgan (1997) extended this work to consider symmetric war of attritions and all-pay auctions: they too focus on symmetric equilibria. Our environment is similar to that discussed in these two papers but we do not restrict to symmetric bidders or symmetric equilibria, and we also extend the analysis to other games. 
There is a number of papers that have attempted a characterization of the equilibrium set for specific asymmetric auction games. None of the following papers treats the combination of common values and reserve price.

(1) First price auction. The first example of nonexistence of equilibrium in two-players first price auctions is in Milgrom and Weber (1985) (footnote 9, p. 625). Lebrun (1997) and Bajari (1996) prove existence of equilibrium in first price auctions with independent private values. Maskin and Riley (1996a) prove existence of equilibrium both for the case in which values are common but signals are independent and for the case in which signals are affiliated but values are private; they do not consider a reserve price. Bajari (1996), Lebrun (1994), and Maskin and Riley (1996b) prove uniqueness of equilibrium with independent private values. Maskin and Riley (1996a, b) allow for risk aversion. None of these papers treats the combination of reserve price and common values.

(2) War of attrition. Nalebuff and Riley (1985) showed that there is a continuum of equilibria in the war of attrition with independent private values. Still in the independent private values setup, Fudenberg and Tirole (1986) assume that in addition to a positive reserve price, some types of each of the two players will "stay in" forever in any equilibrium. They showed that adding this final condition yields uniqueness of equilibrium, because equilibria are ordered in terms of "relative toughness": we borrow this term from them, and extend their intuition to a much larger class of games.

(3) All-pay auctions. Amman and Leininger (1996) prove existence and uniqueness of equilibrium for a class of two bidders all-pay auctions with independent private values. This work is closer to ours because their analysis applies to a class of auctions instead of only one, but it does not cover common values.

(4) Second price and English auction. As mentioned above, Milgrom (1981) shows that in the second price auction with two bidders there is a continuum of equilibria. Bickchandani and Riley (1991) give conditions for uniqueness in the second price auction with more than two bidders, and extend Milgrom's analysis to show multiplicity in the English auction with $n$ bidders.

(5) Existence. Athey (1997) independently proves existence of purestrategy equilibria in a class of $n$-players games. Her setup is similar to ours in that she concentrates on games where each player's payoff satisfies a single crossing condition which is the same as our condition QM. Athey's methodology relies on a continuity assumption that rules out a reserve price.

The novel features of our paper are the following: We study the affiliated interdependent values environment; we allow for reserve prices, something 
that is straightforward in the private values case but presents the difficulties mentioned before in an environment where players' valuations may depend on the signals of their opponents; and we analyze a large class of games together. The last feature is attractive because it unifies the literature by explaining what aspects of the games yield uniqueness and what conditions fail when there is multiplicity. Indeed it turns out that both in the second price auction and in the war of attrition, which have been shown in the literature to exhibit multiple equilibria, the winning bidders' payoff is weakly, but not strictly decreasing in his own bid. This paper shows that "strict decreasingness" guarantees uniqueness of the equilibrium.

\subsection{Structure of the Paper}

In section 2 we introduce the model, some definitions and the main assumptions. We present the concepts of affiliation and quasimonotonicity, and relate the two; these afford the unifying language allowing us to treat our very general model of a bidding game. Section 3 deals with the question of uniqueness. For the analysis of section 3 we assume that strategies are regular in the sense that they can be represented by Lipschitz differential equations and initial conditions: the Appendix gives conditions under which this assumption is legitimate. Section 4 contains our existence result. Section 5 applies our analysis to prove existence and uniqueness of equilibrium for auctions such as: First price, combinations of first price and second price, all-pay auction, combinations of all-pay auction and war of attrition and a first price without risk-neutrality. The Appendix proves the regularity of equilibrium strategies. It first shows that, in the case of independence, strategies have to be monotonic. It then proceeds to prove that monotone equilibrium strategies have to be continuous, strictly increasing, differentiable, and Lipschitz.

\section{SETUP AND DEFINITIONS}

\subsection{Affiliation and Quasimonotonicity}

Here we define the concept of affiliation introduced by Milgrom and Weber (1982), and the concept of quasimonotonicity [closely related to the single-crossing property used in Milgrom and Shannon (1994) and Athey $(1995,1997)]$. We also present a well-known lemma that links the two concepts.

DEFInITIOn 1. Two random variables $\Theta_{i}$ and $\Theta_{j}$ with joint density $f$ are said to be affiliated if

$$
\theta_{i}^{\prime} \geq \theta_{i} \text { and } \theta_{j}^{\prime} \geq \theta_{j} \Rightarrow f\left(\theta_{i}^{\prime}, \theta_{j}^{\prime}\right) f\left(\theta_{i}, \theta_{j}\right) \geq f\left(\theta_{i}^{\prime}, \theta_{j}\right) f\left(\theta_{i}, \theta_{j}^{\prime}\right) .
$$


The property that we introduce next has been used by Milgrom and Shannon (1994) and Athey (1995), but was given no name there; so we use the terminology introduced in Karamardian and Schaible (1990):

Definition 2. We say that a real function $Q(\theta)$ is quasimonotone in $\theta$ if

$$
Q(\theta)>0 \Rightarrow Q\left(\theta^{\prime}\right) \geq 0 \text { for all } \theta^{\prime}>\theta .
$$

We say that a real function $Q(\cdot)$ is strictly quasimonotone if $Q$ is quasimonotone and is almost everywhere different from 0 .

Quasimonotonicity requires that a function only changes sign once, and then from negative to positive, as its argument increases. Quasimonotonicity is instrumental in defining the single-crossing property: A function $u(a, \theta): \mathbb{R}^{2} \rightarrow \mathbb{R}$ is said to have the single-crossing property if, for any given $a^{\prime}>a, u\left(a^{\prime}, \theta\right)-u(a, \theta)$ is quasimonotone in $\theta$ [see Milgrom and Shannon (1994)].

The next lemma connects quasimonotonicity and affiliation (references can be found in Athey, 1995).

Lemma 1. Suppose that $Q\left(\theta_{j}\right)$ is quasimonotone in $\theta_{j}$, that $\Theta_{i}, \Theta_{j}$ are affiliated and $\int Q\left(\theta_{j}\right) f_{j}\left(\theta_{j} \mid \theta_{i}\right) d \theta_{j}=0$. Then $\int Q\left(\theta_{j}\right) f_{j}\left(\theta_{j} \mid \theta_{i}^{\prime}\right) d \theta_{j} \geq 0$ for $\theta_{i}^{\prime} \geq \theta_{i}$.

\subsection{Payoffs}

The payoff function for each player $i$ is

$$
\begin{aligned}
& \pi_{i}^{W}\left(b_{i}, b_{j}, \theta_{i}, \theta_{j}\right) I_{b_{j}<b_{i}}+\pi_{i}^{L}\left(b_{i}, b_{j}, \theta_{i}, \theta_{j}\right) I_{b_{j}>b_{i}} \\
& \quad+\frac{1}{2}\left[\pi_{i}^{W}\left(b_{i}, b_{j}, \theta_{i}, \theta_{j}\right)+\pi_{i}^{L}\left(b_{i}, b_{j}, \theta_{i}, \theta_{j}\right)\right] I_{b_{i}=b_{j}}
\end{aligned}
$$

Types are modeled as random variables $\Theta_{i}(i=1,2)$ with joint density $f\left(\theta_{1}, \theta_{2}\right)$ on $\left[\underline{\theta}_{1}, \bar{\theta}_{1}\right] \times\left[\underline{\theta}_{2}, \bar{\theta}_{2}\right]$. Without loss of generality, we can linearly rescale signals so that both players have the same lower and upper element of the support, i.e. $\theta_{1}=\theta_{2}=\underline{\theta}$ and $\overline{\theta_{1}}=\overline{\theta_{2}}=\bar{\theta}$.

We will denote the distribution of types of player $j$ conditional on type $i$ being $\theta_{i}$ by $f_{j}\left(\cdot \mid \theta_{i}\right)$. We posit that a reserve price $r$ exists, such that a positive mass of types of each player bids under $r$. If a player bids below $r$, he will be "held out of the auction" and will receive 0 payoff. When a player $i$ bids at or above, and his opponent bids below, $r$ the player receives $\pi_{i}^{W}\left(b_{i}, r, \theta_{i}, \theta_{j}\right)$.

There is no difficulty in interpreting the functions $\pi_{i}^{W}\left(b_{i}, b_{j}, \theta_{i}, \theta_{j}\right)$ and $\pi_{i}^{L}\left(b_{i}, b_{j}, \theta_{i}, \theta_{j}\right)$ as expected values of more primitive objects, conditional on $\theta_{i}$ and $\theta_{j}$. This interpretation allows us to subsume, for example, the 
"general affiliated model" presented in Milgrom and Weber (1982). Following are examples of payoff functions we will be concerned with: an object of value $V_{i}\left(\theta_{i}, \theta_{j}\right) \geq 0$ is for sale according to one of the following mechanisms:

EXAMPLE 1. First Price Auction: $\pi_{i}^{W}\left(b_{i}, b_{j}, \theta_{i}, \theta_{j}\right)=V_{i}\left(\theta_{i}, \theta_{j}\right)-b_{i}$ and $\pi_{i}^{L}\left(b_{i}, b_{j}, \theta_{i}, \theta_{j}\right)=0$.

EXAMPLE 2. All-Pay Auction: $\pi_{i}^{W}\left(b_{i}, b_{j}, \theta_{i}, \theta_{j}\right)=V_{i}\left(\theta_{i}, \theta_{j}\right)-b_{i}$ and $\pi_{i}^{L}\left(b_{i}, b_{j}, \theta_{i}, \theta_{j}\right)=-b_{i}$.

EXAMPLE 3. War of Attrition: $\pi_{i}^{W}\left(b_{i}, b_{j}, \theta_{i}, \theta_{j}\right)=V_{i}\left(\theta_{i}, \theta_{j}\right)-b_{j}$ and $\pi_{i}^{L}\left(b_{i}, b_{j}, \theta_{i}, \theta_{j}\right)=-b_{i}$.

EXAMPLE 4. Combination of First and Second Price Auction: $\pi_{i}^{W}\left(b_{i}, b_{j}\right.$, $\left.\theta_{i}, \theta_{j}\right)=V_{i}\left(\theta_{i}, \theta_{j}\right)-\left(\alpha b_{i}+(1-\alpha) b_{j}\right)$ and $\pi_{i}^{L}\left(b_{i}, b_{j}, \theta_{i}, \theta_{j}\right)=0$ with $\alpha \in$ $(0,1)$.

EXAmPLe 5. Combination of All-Pay Auction and War of Attrition: $\pi_{i}^{W}\left(b_{i}, b_{j}, \theta_{i}, \theta_{j}\right)=V_{i}\left(\theta_{i}, \theta_{j}\right)-\left(\alpha b_{i}+(1-\alpha) b_{j}\right)$ and $\pi_{i}^{L}\left(b_{i}, b_{j}, \theta_{i}, \theta_{j}\right)=$ $-b_{i}$ with $\alpha \in(0,1)$.

EXAMPLE 6. First Price Auction with risk aversion: $\pi_{i}^{W}\left(b_{i}, b_{j}, \theta_{i}, \theta_{j}\right)=$ $u_{i}\left(V_{i}\left(\theta_{i}, \theta_{j}\right)-b_{i}\right)$ where $u_{i}(\cdot)$ is strictly increasing, strictly concave and differentiable, and $\pi_{i}^{L}\left(b_{i}, b_{j}, \theta_{i}, \theta_{j}\right)=0$.

Since we are concerned with the common-values aspect of bidding games, we posit that $V_{i}\left(\theta_{i}, \theta_{j}\right)$ is strictly increasing in both arguments.

\subsection{Strategies}

Pure strategies are measurable functions $b_{i}:[\underline{\theta}, \bar{\theta}] \rightarrow(-\infty,+\infty)$.

The profits to player $\theta_{i}$ from playing $b$ when his opponent's strategy is $b_{j}(\cdot)$ will be

$$
\Pi_{i}\left(\theta_{i}, b, b_{j}\right)=\left\{\begin{array}{cl}
\int_{\theta_{j}: b_{j}\left(\theta_{j}\right)<b} \pi_{i}^{W}\left(b, b_{j}\left(\theta_{j}\right) \vee r, \theta_{i}, \theta_{j}\right) f_{j}\left(\theta_{j} \mid \theta_{i}\right) d \theta_{j} \\
\quad+\int_{\theta_{j}: b_{j}\left(\theta_{j}\right)>b} \pi_{i}^{L}\left(b, b_{j}\left(\theta_{j}\right), \theta_{i}, \theta_{j}\right) f_{j}\left(\theta_{j} \mid \theta_{i}\right) d \theta_{j} \\
+\frac{1}{2} \int_{\theta_{j}: b_{j}\left(\theta_{j}\right)=b}\left[\pi_{i}^{W}\left(b, b, \theta_{i}, \theta_{j}\right)+\pi_{i}^{L}\left(b, b, \theta_{i}, \theta_{j}\right)\right] \\
\cdot f_{j}\left(\theta_{j} \mid \theta_{i}\right) d \theta_{j} & \text { for } b \geq r \\
0 & \text { for } b<r
\end{array}\right.
$$


We call regular strategies those pure strategies that are nondecreasing on the whole range, and for bids strictly above $r$ are continuous, strictly increasing, differentiable and Lipschitz continuous.

We now define mixed strategies, using the concept of behavioural strategy. Let $\mathscr{B}$ be the class of Borel subsets of the real line, and let $A \in \mathscr{B}$. The function $\eta_{j}: \mathscr{B} \times[\underline{\theta}, \bar{\theta}] \rightarrow[0,1]$ is a behavioural strategy for player $j$ if

1. $\eta_{j}\left(\cdot, \theta_{j}\right): \mathscr{B} \rightarrow[0,1]$ is a probability measure $\forall \theta_{j} \in[\underline{\theta}, \bar{\theta}]$.

2. $\eta_{j}(A, \cdot):[\underline{\theta}, \bar{\theta}] \rightarrow[0,1]$ is measurable.

DEFINITION 3. A behavioural strategy $\eta_{j}(\cdot, \cdot)$ is nondecreasing if whenever $\theta_{j}^{\prime}>\theta_{j}$, every element of the support of $\eta_{j}\left(\cdot, \theta_{j}^{\prime}\right)$ is greater or equal than every element of the support of $\eta_{j}\left(\cdot, \theta_{j}\right)$.

Let $T \in \mathscr{B} \cap[\underline{\theta}, \bar{\theta}]$. Then we can define

$$
\mu_{j}\left(A, T \mid \theta_{i}\right):=\int_{T} \eta_{j}\left(A, \theta_{j}\right) f_{j}\left(\theta_{j} \mid \theta_{i}\right) d \theta_{j}
$$

to be the probability measure on the space of actions and types of player $j$ induced by the behavioural strategy $\eta_{j}$, in $\theta_{i}$ 's opinion. Analogously, we define

$$
\mu_{j}(A, T):=\int_{T} \eta_{j}\left(A, \theta_{j}\right) f_{j}\left(\theta_{j}\right) d \theta_{j} .
$$

Now, we can also denote

$$
\mu_{j}\left(A \mid \theta_{i}\right):=\mu_{j}\left(A,[\underline{\theta}, \bar{\theta}] \mid \theta_{i}\right),
$$

the marginal distribution of bids induced by the strategy $\eta_{j}$ in $\theta_{i}$ 's opinion, and similarly

$$
\mu_{j}(A):=\mu_{j}(A,[\underline{\theta}, \bar{\theta}]) .
$$

The payoff to type $\theta_{i}$ of bidding $b \geq r$ when his opponent plays according to $\eta_{j}$ is

$$
\begin{aligned}
& \Pi_{i}\left(\theta_{i}, b, \eta_{j}\right) \\
& =\int_{(-\infty, b) \times[\underline{\theta}, \bar{\theta}]} \pi_{i}^{W}\left(b, s \vee r, \theta_{i}, \theta_{j}\right) \mu_{j}\left(d s, d \theta_{j} \mid \theta_{i}\right) \\
& \quad+\int_{(b,+\infty) \times[\underline{\theta}, \bar{\theta}]} \pi_{i}^{L}\left(b, s, \theta_{i}, \theta_{j}\right) \mu_{j}\left(d s, d \theta_{j} \mid \theta_{i}\right) \\
& \quad+\frac{1}{2} \int_{\{b\} \times[\underline{\theta}, \bar{\theta}]}\left[\pi_{i}^{W}\left(b, s \vee r, \theta_{i}, \theta_{j}\right)+\pi_{i}^{L}\left(b, s, \theta_{i}, \theta_{j}\right)\right] \mu_{j}\left(d s, d \theta_{j} \mid \theta_{i}\right) .
\end{aligned}
$$

Denote $B_{j}=\sup \left\{b: b \in \operatorname{support}\left(\mu_{j}(\cdot)\right)\right\},{ }^{1}$ and let $B=B_{1} \vee B_{2}$. Hence, $B$ is the maximum bid that any of the players will ever play.

\footnotetext{
${ }^{1}$ In the case of pure strategies, this definition reduces to $B_{j}=\sup _{\theta_{j}} b_{j}\left(\theta_{j}\right)$.
} 


\subsection{Assumptions}

We now introduce and motivate the key assumptions used in the paper. The first two assumptions specify the statistical structure of the model.

A1. $f(\cdot, \cdot)$ is of class $C^{1}$ and $f\left(\theta_{1}, \theta_{2}\right)>0$ on $(\underline{\theta}, \bar{\theta}] \times(\underline{\theta}, \bar{\theta}]$.

A2. $\Theta_{1}, \Theta_{2}$ are affiliated.

Assumption $\mathbf{A 2}$ imposes a monotone structure on conditional expectations. Next come assumptions on the payoff functions, i.e. the rules of the game.

A3. $\Pi_{i}\left(\underline{\theta}, b, \eta_{j}\right)<0$ for all $i, b \geq r, \eta_{j}$.

Assumption $\mathbf{A 3}$ is a requirement on the reserve price $r$ : It ensures that there is a set of types of both players that does not find it convenient to bid actively, no matter what their opponent does. Any condition that guarantees this is sufficient for our results, and this assumption is reasonable when the auctioneer sets a reserve price. In a first price auction, all-pay auction and war of attrition, it means that the expected value of the object conditional on being the lowest type is less than the reserve price.

A4. $\pi_{i}^{W}\left(b_{i}, b_{j}, \theta_{i}, \theta_{j}\right)$ and $\pi_{i}^{L}\left(b_{i}, b_{j}, \theta_{i}, \theta_{j}\right)$ are continuously differentiable.

A5. $\pi_{i}^{L}\left(b_{i}, b_{j}, \theta_{i}, \theta_{j}\right)$ is nonpositive.

A6. $\pi_{i}^{W}\left(b_{i}, b_{j}, \theta_{i}, \theta_{j}\right)$ is strictly increasing in $\theta_{i}$ and $\theta_{j}$; moreover $\pi_{i}^{L}\left(b_{i}, b_{j}, \theta_{i}, \theta_{j}\right)$ is nondecreasing in $\theta_{i}$.

Assumption $\mathbf{A 6}$ says that being a high type is good for your payoff, and it is better to win against a high than against a low type: this is an essential feature of the common value model.

A7. $\pi_{i}^{W}\left(b_{i}, b_{j}, \theta_{i}, \theta_{j}\right)$ is strictly decreasing in $b_{i}$ and there is a $\overline{b_{i}}$ such that $\pi_{i}^{W}\left(\overline{b_{i}}, b_{j}, \theta_{i}, \theta_{j}\right)<0$ for any choice of $b_{j}, \theta_{i}, \theta_{j} ;$ moreover, $\pi_{i}^{L}\left(b_{i}, b_{j}, \theta_{i}, \theta_{j}\right)$ is nonincreasing in $b_{i}$ and does not depend on $b_{j}$.

Assumption A7 says that, conditional on winning (or losing) the object, bidding less is always preferred and that there is a maximum bid at which even winning is unprofitable. The payoff when losing does not depend on the winning bid. The first part of assumption $\mathbf{A} 7$ is crucial to the interpretation of this paper: indeed, both in the second-price auction and in the war of attrition $\pi_{i}^{W}$ fails to be strictly decreasing in $b_{i}$. This has the consequence that both mechanisms are "uniqueness challenged." 
A8. $\pi_{i}^{W}\left(b_{i}, b_{j}, \theta_{i}, \theta_{j}\right) I_{\theta_{j}<\psi_{j}}+\pi_{i}^{L}\left(b_{i}, b_{j}, \theta_{i}, \theta_{j}\right) I_{\theta_{j}>\psi_{j}}$ is quasimonotone in $\theta_{j}$.

Assumption A8 will be used in Proposition 1 to characterize the set of admissible initial conditions; the lowest actively bidding types. Since $\pi_{i}^{W}\left(b_{i}, b_{j}, \theta_{i}, \theta_{j}\right)$ is increasing in $\theta_{j}$ (by $\mathbf{A 6}$ ), assumption $\mathbf{A 8}$ is verified whenever $\pi_{i}^{L}\left(b_{i}, b_{j}, \theta_{i}, \theta_{j}\right)$ is 0 .

All assumptions pertaining to the "rules of the game" are verified in examples 1-6, with the exception of $\mathbf{A} 7$ which fails in example 3: we will later expand on the significance of this observation.

For expositional purposes, the results of the next section rely on the assumption that strategies are regular and on an additional requirement on the first-order conditions (condition (QM) in section 3.3). In the Appendix we give sufficient conditions for behavioural strategy equilibria to be in regular strategies. Condition (QM) is proved to hold for all regular strategy equilibria in each game we consider in section 5 .

\section{UNIQUENESS}

We are aiming at a characterization of equilibrium inverse bidding functions as the solution to a system of differential equations plus a set of initial conditions.

Assume $b_{i}, b_{j}:[\underline{\theta}, \bar{\theta}] \rightarrow \Re$ are regular equilibrium strategies, hence strictly increasing whenever the bid is higher than $r$ : we can define the inverse bidding functions $\phi_{i}(\cdot)$ as

$$
\phi_{i}:\left(r, B_{i}\right] \rightarrow[\underline{\theta}, \bar{\theta}], \quad \phi_{i}(\cdot)=b_{i}^{-1}(\cdot) .
$$

Let the initial conditions $\theta_{i}^{0}$ be defined by

$$
\theta_{i}^{0}=\lim _{b \downarrow r} \phi_{i}(b) .
$$

In view of $\mathrm{A} 3, \theta_{i}^{0}>\underline{\theta}$.

Because regular strategies are Lipschitz, a unique trajectory originates from each initial condition [see Hirsch and Smale (1974), p. 162]; so to prove uniqueness of equilibrium it suffices to show that there cannot be two equilibria starting from two different initial conditions. This result stems from the examination of the inverse bidding functions, as represented in Figure 1, and proceeds in three steps. 


\subsection{The "Relative Toughness" Logic of the Uniqueness Result}

The first step (initial conditions) is making sure that the possible initial conditions are ordered in terms of relative toughness: This is done in subsection 3.2. The idea is that winning against a high type is better, so when many types of player 2 are staying out of the auction it pays even for a relatively low type of player 1 to enter, since he will be winning against high types of the opponent; thus a very aggressive behaviour on the part of player $i$ corresponds to an accommodating behaviour by player $j$. In terms of Figure 1, if two pairs of initial conditions $\left(\theta_{1}^{0}, \theta_{2}^{0}\right)$ and $\left(\widehat{\theta}_{1}^{0}, \widehat{\theta}_{2}^{0}\right)$ are candidate for being part of two (coexisting) equilibria and $\theta_{1}^{0}<\widehat{\theta}_{1}^{0}$, then $\theta_{2}^{0}>\widehat{\theta}_{2}^{0}$; graphically, one pair is "nested" into the other.

The second step (final condition) is that, whenever the payoff conditional on winning is decreasing in one's own bid, there is a "final condition" to be met by an equilibrium, in that the highest types of both players must bid the same amount. This is because otherwise it would be convenient for one of them to decrease his bid (this reasoning assumes that the highest bidder bids a finite amount, and assumption A7 above guarantees this). In terms of Figure 1, in each equilibrium the trajectories departing from the initial conditions must meet at the endpoint (recall we are dealing with regular, hence strictly increasing, strategies), although this endpoint may be different for different equilibria.

The third step (no crossing) concludes the proof by noting that for two equilibria to coexist what would have to happen is that, for at least one player, the trajectories describing the two equilibria must cross [this is best understood by looking at Figure 1: for $\widehat{\phi}_{1}(\cdot)$ to meet $\widehat{\phi}_{2}(\cdot)$ at the endpointstep 2 earlier-given the way in which initial conditions are nested (step 1) it has to be that either $\widehat{\phi}_{1}(\cdot)$ crosses $\phi_{1}(\cdot)$ or $\widehat{\phi}_{2}(\cdot)$ crosses $\left.\phi_{2}(\cdot)\right]$. But subsection 3.3 rules this out, again resorting to an argument of relative toughness. Take the lowest bid at which there is a crossing ( $b^{*}$ in Figure 1),

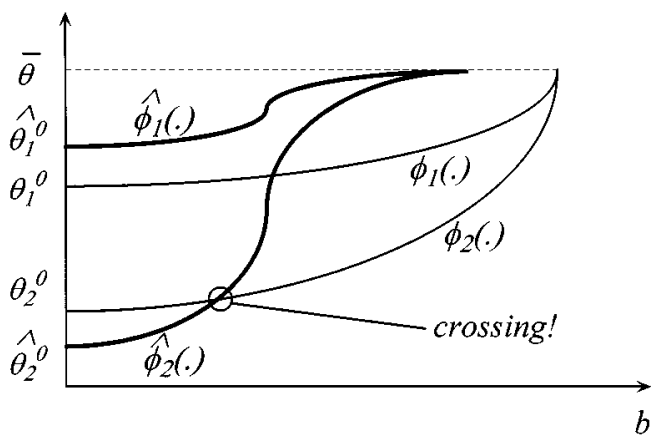

FIG. 1. Inverse bidding functions. 
and consider the opponent in the " $\phi$ equilibrium" [type $\left.\phi_{1}\left(b^{*}\right)\right]$ of the player whose trajectories cross: in the " $\widehat{\phi}$ equilibrium" this type could-by bidding $b^{*}$-be facing the same opponent he was bidding against in the " $\phi$ equilibrium," who bids the same as in the " $\phi$ equilibrium," but on the margin is less aggressive. Yet type $\phi_{1}\left(b^{*}\right)$ chooses to play less aggressively than in the " $\phi$ equilibrium" (he bids less), which is inconsistent with relative toughness because he should be behaving more aggressively.

This shows that there cannot be two equilibria in regular strategies.

\subsection{Initial Conditions}

This subsection characterizes the set of possible initial conditions for equilibrium inverse bidding functions. In Lemma 2 we find the locus of points (curves) in the space of types, that are possible initial conditions; Propositions 1 and 2 give conditions under which these curves are negatively sloped, or in other words they are ranked by "relative toughness." The concept of "relative toughness" is formalized as " 0 substitutes" in Definition 4.

Let us characterize the pair $\left(\theta_{1}^{0}, \theta_{2}^{0}\right)$ defined in (3). Define

$$
\begin{aligned}
H_{i}^{r}\left(\psi_{i}, \psi_{j}\right):= & \int_{-\infty}^{\psi_{j}} \pi_{i}^{W}\left(r, r, \psi_{i}, \theta_{j}\right) f_{j}\left(\theta_{j} \mid \psi_{i}\right) d \theta_{j} \\
& +\int_{\psi_{j}}^{+\infty} \pi_{i}^{L}\left(r, b_{j}\left(\theta_{j}\right), \psi_{i}, \theta_{j}\right) f_{j}\left(\theta_{j} \mid \psi_{i}\right) d \theta_{j} .
\end{aligned}
$$

Under A7, this expression does not depend on the equilibrium strategy $b_{j}(\cdot)$. This is the expected payoff of a player $\psi_{i}$ who plays $r+\varepsilon$ for $\varepsilon$ arbitrarily small, when the opponent's highest type bidding $r$ is $\psi_{j}$.

Lemma 2. Assume A3, A4, and A7. An equilibrium pair $\left(\theta_{1}^{0}, \theta_{2}^{0}\right)$ satisfies $H_{i}^{r}\left(\theta_{i}^{0}, \theta_{j}^{0}\right) \geq 0$ for all $i, j$ and equality holds for $i, j$ or both.

Proof. First, observe that in equilibrium it must be true that

$$
H_{i}^{r}\left(\theta_{i}^{0}, \theta_{j}^{0}\right) \geq 0 \text { for } i \neq j, \quad i, j=1,2 .
$$

Indeed, suppose this was not the case for type $\theta_{i}^{0}$, say, i.e. the first inequality in (6) does not hold; then there would be a $\theta_{i}^{\prime}$ slightly greater than $\theta_{i}^{0}$ who, by definition of $\theta_{i}^{0}$ is bidding actively, and by $\mathbf{A 4}$ is getting an expected payoff arbitrarily close to $H_{i}^{r}\left(\theta_{i}^{0}, \theta_{j}^{0}\right)$, which would be negative. But this cannot be, since then $\theta_{i}^{\prime}$ would better off not bidding.

Let us now observe, to finish the proof, that in equilibrium it cannot be that both inequalities in (6) hold strictly. If this was the case, by $\mathbf{A 3}$ there 
would exist types $\tilde{\theta}_{1}<\theta_{1}^{0}$ and $\tilde{\theta}_{2}<\theta_{2}^{0}$ whose bid is equal to $r$ in view of nondecreasing strategies (bidding below $r$ gives 0 payoff). This implies that there would be a mass of player 1 types and a mass of player 2 types both bidding $r$, and this is impossible in a nondecreasing strategies equilibrium, because one of the two players will find it profitable to deviate.

Remark. The previous result rules out the possibility that players tie at $r$ with positive probability. It is possible that $H_{i}^{r}\left(\theta_{i}^{0}, \theta_{j}^{0}\right)>0$ for some $i$, and thus that a mass of types of one of the players bids $r$ (see Lemma 3 and the discussion preceding it). This cannot happen for bids bigger than $r$, as proved in Lemma 6 of the Appendix.

We now define the notion of 0 substitutes, which formalizes our intuition of "relative aggressiveness": the idea is that given a function of two variables that has value of 0 at some point, increasing one variable requires a decrease in the other in order to keep the level of the function at 0 . In other words, the level curves of the function at 0 are negatively sloped.

Definition 4. Given a function $F(x, y): \Re^{2} \rightarrow \Re$, we say that $x$ is a $\mathbf{0}$ substitute of $y$ in $F$ when

$$
F(x, y)=0=F(\hat{x}, \hat{y}) \text { and } \hat{x}>x \text { imply } \hat{y}<y .
$$

Our objective is now to show that the level curves $H_{i}^{r}\left(\psi_{i}, \psi_{j}\right)=0$ are negatively sloped, or equivalently that $H$ is increasing in its argument at 0 ; this is the import of the next two propositions.

Notice that $H$ is obtained integrating the expression in $\mathbf{A 8}$ with respect to the distribution of types of player $j$ conditional on $\psi_{i}$. So it is easy to understand why assumption $\mathbf{A 8}$ is important for $H$ to be increasing in $\psi_{i}$ : as player $i$ 's type increases, along with a positive effect on the payoff functions $\pi_{i}$, he becomes more optimistic about his opponent's type distribution (this effect is of course absent when types are independent). This entails a higher probability of losing with a given bid. When losing is costly (which A8 rules out), this negative effect may offset the previous positive one.

Proposition 1. Assume A5, A6, A7, and, either $\mathbf{A 2}$ and $\mathbf{A 8}$, or that $\Theta_{i}$, $\Theta_{j}$ are independent. Then $\psi_{j}$ is a 0 substitute of $\psi_{i}$ in $H_{i}^{r}\left(\psi_{i}, \psi_{j}\right)$.

Proof. It is sufficient to show that $H$ is strictly quasimonotone in $\psi_{i}$ and in $\psi_{j}$, since this means that the level curves $H=0$ have negative slope in the $\psi_{i} \times \psi_{j}$ plane. To prove that $H$ is strictly quasimonotone in $\psi_{i}$ observe that by $\mathbf{A 6}$ the $\pi$ terms in (4) are strictly increasing in $\psi_{i}$, so it suffices to show that the change in the probability term $f_{j}\left(\cdot \mid \psi_{i}\right)$, as $\psi_{i}$ increases does not reduce the expression. If $\Theta_{i}, \Theta_{j}$ are independent there is no change, while under affiliation the change is nonnegative if $\mathbf{A 8}$ is verified (see Lemma 1 in section 2.1). 
Second, to prove that $H$ is strictly quasimonotone in $\psi_{j}$ it suffices to show that when $H=0$, then its derivative with respect to $\psi_{j}$ is positive, i.e. $\pi_{i}^{W}\left(r, r, \psi_{i}, \psi_{j}\right)-\pi_{i}^{L}\left(r, r, \psi_{i}, \psi_{j}\right)>0$. Since $\pi_{i}^{L} \leq 0$ by $\mathbf{A 5}$, for $H_{i}^{r}\left(\psi_{i}, \psi_{j}\right)$ to equal 0 it has to be that $\int_{-\infty}^{\psi_{j}} \pi_{i}^{W}\left(r, r, \psi_{i}, \theta_{j}\right) f_{j}\left(\theta_{j} \mid \psi_{i}\right) d \theta_{j}>0$ whence, since $\pi_{i}^{W}$ is increasing in $\theta_{j}$ by $\mathbf{A 6}, \pi_{i}^{W}\left(r, r, \psi_{i}, \psi_{j}\right)>0>\pi_{i}^{L}\left(r, r, \psi_{i}, \psi_{j}\right)$.

The above proposition, while completely satisfactory in the case of independent signals, has limited applicability when signals are not independent because of A8. This assumption rules out games where the loser pays, like the war of attrition or the all-pay auction, and the difficulty arising in those cases has been explained. It is nonetheless possible to give quite general conditions under which we can analyze those games: this is done in the next proposition.

Proposition 2. Assume A2, and that the payoff in (1) can be written as:

$$
\left[V_{i}\left(\theta_{i}, \theta_{j}\right)-C_{i}^{W}\left(b_{i}, b_{j}\right)\right] I_{b_{j}<b_{i}}-C_{i}^{L}\left(b_{i}\right) I_{b_{i}<b_{j}},
$$

where $V$ is increasing in both arguments, $V_{i}\left(\theta_{i}, \theta_{j}\right) f_{j}\left(\theta_{j} \mid \theta_{i}\right)$ is increasing in $\theta_{i}$, $C_{i}^{W}\left(b_{i}, b_{j}\right)$ is strictly increasing in $b_{i}, C_{i}^{L}$ is nonnegative, $C_{i}^{W}(r, r) \geq C_{i}^{L}(r) \geq$ 0 . Then $\psi_{j}$ is a 0 substitute of $\psi_{i}$ in $H_{i}^{r}\left(\psi_{i}, \psi_{j}\right){ }^{2}$

Proof. Again, it suffices to show that $H_{i}^{r}\left(\psi_{i}, \psi_{j}\right)$ is strictly quasimonotone in $\psi_{i}$ and in $\psi_{j} . H_{i}$ is strictly quasimonotone in $\psi_{j}$ by the same argument as in Proposition 1. Under the assumptions above we have:

$$
\begin{aligned}
H_{i}^{r}\left(\psi_{i}, \psi_{j}\right)= & \int_{-\infty}^{\psi_{j}} V_{i}\left(\psi_{i}, \theta_{j}\right) f_{j}\left(\theta_{j} \mid \psi_{i}\right) d \theta_{j} \\
& -\left(C_{i}^{w}(r, r)-C_{i}^{l}(r)\right) F\left(\psi_{j} \mid \psi_{i}\right)-C_{i}^{l}(r)
\end{aligned}
$$

Because $V_{i}\left(\psi_{i}, \theta_{j}\right) f_{j}\left(\theta_{j} \mid \psi_{i}\right)$ is increasing in $\psi_{i}$ the integral is increasing in $\psi_{i}$. By affiliation, $F\left(\psi_{j} \mid \psi_{i}\right)$ decreases in $\psi_{i}$ so that the second term increases in $\psi_{i}$.

Lemma 2 above told us that the possible initial conditions for equilibrium inverse bidding functions lie on the "North-East envelope" of the level curves $H_{i}^{r}\left(\psi_{i}, \psi_{j}\right)=0$ (the thick line in Figure 2). Note that the level curve $H_{1}^{r}\left(\psi_{1}, \psi_{2}\right)=0$ will cross the perimeter of the $[\underline{\theta}, \bar{\theta}]^{2}$ square somewhere in the interior of the top edge, because by assumption $\mathbf{A 3}$ type $\underline{\theta}$ strictly

\footnotetext{
${ }^{2}$ The assumption that $V_{i}\left(\theta_{i}, \theta_{j}\right) f_{j}\left(\theta_{j} \mid \theta_{i}\right)$ is increasing in $\theta_{i}$ is a special case (the case of $\alpha=1$ ) of assumption A9 (introduced in section 5). Because assumption A9 holds for $\alpha^{\prime}$ if it holds for any $\alpha<\alpha^{\prime}$, the condition that guarantees that the Nort-East envelope is negatively sloped is weaker than the condition that guarantees that there exists an equilibrium in nondecreasing strategies.
} 


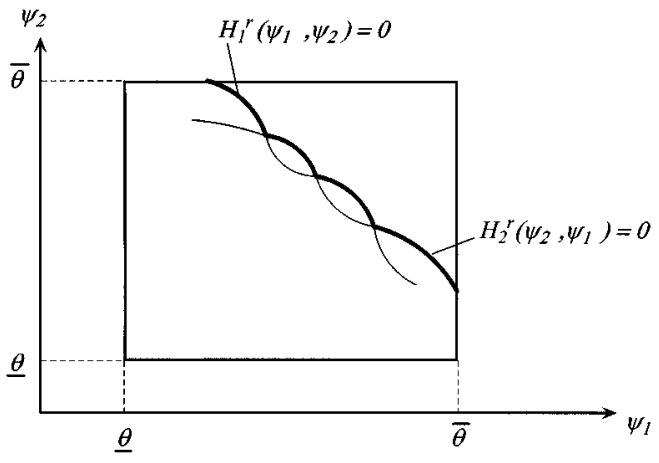

FIG. 2. North-East envelope. The possible initial conditions lie on the thickened line.

prefers not to bid, hence $H_{1}^{r}\left(\underline{\theta}, \psi_{2}\right)<0$ for all $\psi_{2}$. Similarly, the curve $H_{2}^{r}\left(\psi_{2}, \psi_{1}\right)=0$ will cross the perimeter of the $[\underline{\theta}, \bar{\theta}]^{2}$ square somewhere in the interior of the right edge. Propositions 1 and 2 gave sufficient conditions for the level curves to have negative slope. As a consequence, the North-East envelope itself will have negative slope, i.e. the possible initial conditions are ranked by "relative toughness."

\subsection{No crossing}

This subsection contains two results: The first verifies that $\phi_{i}$ is a 0 substitute of $\phi_{j}^{\prime}$ in the first order conditions for player $i$; the second uses this property to guarantee that trajectories starting on the North-East envelope cannot cross.

At a Nash equilibrium, the first order conditions must be verified, hence

$$
F O C_{i}\left(\phi_{j}^{\prime}(b), \phi_{j}(b), \theta_{i}, b\right):=\left.\frac{\partial}{\partial b} \Pi_{i}\left(\theta_{i}, b, b_{j}(\cdot)\right)\right|_{b=b_{i}\left(\theta_{i}\right)}=0 \text { for } i=1,2 .
$$

Next is a condition that is not given on primitives. It is a standard requirement used to guarantee the existence of equilibria in increasing strategies, and as such is verified in all — to the best of our knowledge-cases of known auction games that display equilibria in increasing strategies. In section 5 we show that $(\mathbf{Q M})$ holds for all equilibria in regular strategies of all the games we consider. in $\theta_{i}$.

(QM) The functions $\operatorname{FOC}_{i}\left(\phi_{j}^{\prime}(b), \phi_{j}(b), \theta_{i}, b\right)$ are quasimonotone

This condition is equivalent to the Single Crossing Condition 2.2 in Athey (1997). 
Proposition 3. Assume A4, A7. If condition (QM) is verified, $\theta_{i}$ is a 0 substitute of $\phi_{j}^{\prime}(b)$ in $\mathrm{FOC}_{i}\left(\phi_{j}^{\prime}(b), \phi_{j}(b), \theta_{i}, b\right)$.

Proof. Since differentiability is guaranteed by $\mathbf{A} 4$ together with the fact that strategies are regular, we can write

$$
\begin{aligned}
F_{i}\left(\phi_{j}^{\prime}(b), \phi_{j}(b), \theta_{i}, b\right) \\
=\left.\frac{\partial}{\partial b} \Pi_{i}\left(\theta_{i}, b, b_{j}(\cdot)\right)\right|_{b=b_{i}\left(\theta_{i}\right)} \\
=\phi_{j}^{\prime}(b) f_{j}\left(\phi_{j}(b) \mid \theta_{i}\right)\left[\pi_{i}^{W}\left(b, b, \theta_{i}, \phi_{j}(b)\right)-\pi_{i}^{L}\left(b, b, \theta_{i}, \phi_{j}(b)\right)\right] \\
\quad+\int_{-\infty}^{\phi_{j}(b)} \frac{\partial}{\partial b} \pi_{i}^{W}\left(b, b_{j}\left(\theta_{j}\right) \vee r, \theta_{i}, \theta_{j}\right) d f_{j}\left(\theta_{j} \mid \theta_{i}\right) \\
\quad+\int_{\phi_{j}(b)}^{+\infty} \frac{\partial}{\partial b} \pi_{i}^{L}\left(b, b_{j}\left(\theta_{j}\right), \theta_{i}, \theta_{j}\right) d f_{j}\left(\theta_{j} \mid \theta_{i}\right) .
\end{aligned}
$$

To check that $\theta_{i}$ is a 0 substitute of $\phi_{j}^{\prime}(b)$, take $\widehat{\theta}_{i}>\theta_{i}$ and $\widehat{\phi}_{j}^{\prime}(b)$ such that $F O C_{i}\left(\widehat{\phi}_{j}^{\prime}(b), \phi_{j}(b), \widehat{\theta}_{i}, b\right)=F O C_{i}\left(\phi_{j}^{\prime}(b), \phi_{j}(b), \theta_{i}, b\right)=0$ : We need to show that $\widehat{\phi}_{j}^{\prime}(b)<\phi_{j}^{\prime}(b)$.

Since the above expression equals 0 at $\left(\theta_{i}, \phi_{j}^{\prime}(b)\right)$, condition (QM) guarantees that increasing $\theta_{i}$ to $\widehat{\theta}_{i}$ makes it positive. Also, observe that the last line in expression (8) is negative at $\left(\widehat{\theta}_{i}, \phi_{j}^{\prime}(b)\right)$ by $\mathbf{A} 7$, and so the coefficient of $\phi_{j}^{\prime}(b)$ must be positive for the expression to be positive at $\left(\widehat{\theta}_{i}, \phi_{j}^{\prime}(b)\right)$. Since $F O C_{i}$ must be 0 at $\left(\widehat{\theta}_{i}, \widehat{\phi}_{j}^{\prime}(b)\right)$ it has to be that $\widehat{\phi}_{j}^{\prime}(b)<\phi_{j}^{\prime}(b)$.

A version of this remarkable property was found to hold in Fudenberg and Tirole (1986) in a war-of-attrition with independent private values.

Setting $\theta_{i}=\phi_{i}(b)$, we can rewrite (8) as a system of differential equations,

$$
F O C_{i}\left(\phi_{j}^{\prime}(b), \phi_{j}(b), \phi_{i}(b), b\right)=0 \text { for } i=1,2 .
$$

This system, together with a pair of initial conditions, will describe each equilibrium pair of trajectories $\left(\phi_{1}(b), \phi_{2}(b)\right)$. Take two pairs of initial conditions $\theta^{0}$ and $\hat{\theta}^{0}$ on the North-East envelope, and let $\phi(\cdot)$ and $\hat{\phi}(\cdot)$ denote the trajectories starting from these initial conditions and described by the system of differential equations (9).

Proposition 4 (No crossing). Suppose $\phi_{i}(b)$ is a 0 substitute of $\phi_{j}^{\prime}(b)$ in $F O C_{i}$ for all $i, b$. Then for all $i, \phi_{i}(\cdot)$ and $\hat{\phi}_{i}(\cdot)$ cannot cross.

Proof. Given two trajectories $\phi_{i}(\cdot)$ and $\hat{\phi}_{i}(\cdot)$ starting from $\theta^{0}$ and $\hat{\theta}^{0}$, let $b^{*}$ be the lowest bid at which $\phi_{i}(\cdot)=\hat{\phi}_{i}(\cdot)$ for some $i$. Assume without loss of generality that for $b<b^{*}$ we have $\hat{\phi}_{1}(b)>\phi_{1}(b)$ and hence (since initial 
conditions lie on the North-East envelope) $\hat{\phi}_{2}(b)<\phi_{2}(b)$. Suppose by contradiction that $\hat{\phi}_{2}\left(b^{*}\right)=\phi_{2}\left(b^{*}\right)$ : it cannot be that also $\hat{\phi}_{1}\left(b^{*}\right)=\phi_{1}\left(b^{*}\right)$ since otherwise integrating backwards the two trajectories would coincide forever (here we use Lipschitz continuity). So it must be that $\hat{\phi}_{1}\left(b^{*}\right)>$ $\phi_{1}\left(b^{*}\right)$ and, by 0 substitutes, $\hat{\phi}_{2}^{\prime}\left(b^{*}\right)<\phi_{2}^{\prime}\left(b^{*}\right)$. But this contradicts the fact that $\hat{\phi}_{2}$ crosses $\phi_{2}$ from below at $b^{*}$.

\section{EXISTENCE}

In order to prove existence, it is not enough to show that a solution to the system of differential equations exists. Indeed, the final condition has to be met: Equilibrium strategies must also "arrive together." In other words, an equilibrium, besides solving (2) and (9) for a suitable set of initial conditions, must have the property that $\phi_{1}(b)=\bar{\theta}$ and $\phi_{2}(b)=\bar{\theta}$ for the same $b$. So the idea of the proof, as executed in Proposition 5, is very simple; start on the upper-left corner of the North-East envelope, and consider the trajectories originating from there and described by (9). By the choice of the initial conditions, the first trajectory to "arrive" will be $\phi_{2}(\cdot)$. Now move along the North-East envelope, down towards the lower-right corner: There, the first trajectory to arrive will be $\phi_{1}(\cdot)$. In between there must be a pair of initial conditions on the North-East envelope where the two trajectories arrive together, and this will be our equilibrium.

In order to make this reasoning go through, however, we first need to check that all points on the North-East envelope are "acceptable" initial conditions for the equilibrium we want to construct. We will call candidate initial conditions for some trajectories, a pair $\theta_{1}^{0}, \theta_{2}^{0}$ such that: all types below them are happy not to bid above $r$, whenever the bidding above $r$ is described by those trajectories; and all types above them are earning nonnegative revenues.

Lemma 3. Let $\phi_{i}(\cdot), \phi_{j}(\cdot)$ be a pair of trajectories solving (9). Assume condition (QM) holds and that for all $i, H_{i}^{r}\left(\psi_{i}, \psi_{j}\right)$ is quasimonotone in $\psi_{i}$. Then all points of the North-East envelope of the level curves $H_{1}^{r}=0, H_{2}^{r}=0$ are candidate initial conditions for the trajectories $\phi_{i}(\cdot), \phi_{j}(\cdot)$.

Proof. If we write $\phi_{i}\left(\theta_{1}^{0}, \theta_{2}^{0}, b\right)$ to denote the trajectory solving (2) and (9), we can define

$$
\bar{b}\left(\theta_{1}^{0}, \theta_{2}^{0}\right):=\min \left\{b: \phi_{1}\left(\theta_{1}^{0}, \theta_{2}^{0}, b\right)=\bar{\theta} \text { or } \phi_{2}\left(\theta_{1}^{0}, \theta_{2}^{0}, b\right)=\bar{\theta}\right\} .
$$

The function $\bar{b}\left(\theta_{1}^{0}, \theta_{2}^{0}\right)$ may be seen as the "hitting time" of the first, among the two trajectories starting from $\left(\theta_{1}^{0}, \theta_{2}^{0}\right)$, to reach the upper bound of its range. 
For a given pair $\theta_{1}^{0}, \theta_{2}^{0}$, let us consider a type $\theta_{i}^{\prime}<\theta_{i}^{0}$. Choose any $b \in$ $\left(r, \bar{b}\left(\theta_{1}^{0}, \theta_{2}^{0}\right)\right)$ and a $\theta_{i}>\theta_{i}^{0}$ so that $b_{i}\left(\theta_{i}\right)=b$. By quasimonotonicity of the first order conditions in $\theta_{i}$ [here we use (QM)], the derivative of $\theta_{i}^{\prime}$ 's payoff at $b$ is negative so this type does not want to bid $b$.

Consider on the other hand a type $\theta_{i}^{\prime}>\theta_{i}^{0}$. This type can bid $r$ and guarantee himself an expected payoff of $H_{i}^{r}\left(\theta_{1}^{\prime}, \theta_{2}^{0}\right)$ which-since $H_{i}^{r}$ is quasimonotone in its arguments-is greater than $H_{i}^{r}\left(\theta_{1}^{0}, \theta_{2}^{0}\right) \geq 0$.

Proposition 5. An equilibrium exists, and is described by (9) and (5) if:

- condition (QM) holds, and

- for all $i, H_{i}^{r}\left(\psi_{i}, \psi_{j}\right)$ is quasimonotone in $\psi_{i}$.

Proof. First, it is clear that the function $\bar{b}\left(\theta_{1}^{0}, \theta_{2}^{0}\right)$ defined in the proof of Lemma 3 is continuous, since the functions $\phi_{i}\left(\theta_{1}^{0}, \theta_{2}^{0}, b\right)$ are continuous in their first two arguments [see Hirsch and Smale (1974)].

Our problem, in view of Lemma 3, reduces to finding a pair of initial conditions $\left(\theta_{1}^{0}, \theta_{2}^{0}\right)$ on the North-East envelope such that

$$
\phi_{1}\left(\theta_{1}^{0}, \theta_{2}^{0}, \bar{b}\left(\theta_{1}^{0}, \theta_{2}^{0}\right)\right)=\bar{\theta} \quad \text { and } \quad \phi_{2}\left(\theta_{1}^{0}, \theta_{2}^{0}, \bar{b}\left(\theta_{1}^{0}, \theta_{2}^{0}\right)\right)=\bar{\theta} .
$$

So, consider the function

$$
I\left(\theta_{1}^{0}, \theta_{2}^{0}\right):=\left[\bar{\theta}-\phi_{1}\left(\theta_{1}^{0}, \theta_{2}^{0}, \bar{b}\left(\theta_{1}^{0}, \theta_{2}^{0}\right)\right)\right]+\left[\phi_{2}\left(\theta_{1}^{0}, \theta_{2}^{0}, \bar{b}\left(\theta_{1}^{0}, \theta_{2}^{0}\right)\right)-\bar{\theta}\right] .
$$

We are looking for zeros of this function lying on the North-East envelope.

Observe that, for points on the North-East envelope with $\theta_{1}^{0}$ close enough to $\bar{\theta}, I\left(\theta_{1}^{0}, \theta_{2}^{0}\right)$ is negative, since $\phi_{2}^{\prime}<\infty$ and $\phi_{1}^{\prime}>0$. For points on the North-East envelope with $\theta_{2}^{0}$ close enough to $\bar{\theta}, I\left(\theta_{1}^{0}, \theta_{2}^{0}\right)$ is positive, reasoning symmetrically.

Also, it is clear that $I(\cdot, \cdot)$ is a continuous function, because of continuity of trajectories with respect to initial conditions [see Hirsch and Smale (1974)].

But then, there is a point $\left(\tilde{\theta}_{1}^{0}, \tilde{\theta}_{2}^{0}\right)$ on the North-East envelope such that

$$
I\left(\tilde{\theta}_{1}^{0}, \tilde{\theta}_{2}^{0}\right)=0 .
$$

Then $\left(\tilde{\theta}_{1}^{0}, \tilde{\theta}_{2}^{0}\right)$ satisfies (5), and together with (3) and (9) characterizes an equilibrium for the auction. To see this, consider that in view of Lemma 3 above, our assumptions guarantee that all points of the North-East envelope of the level curves $H_{1}^{r}=0, H_{2}^{r}=0$ are candidate initial conditions for the trajectories $\phi_{i}(\cdot), \phi_{j}(\cdot)$. So all types below $\left(\tilde{\theta}_{1}^{0}, \tilde{\theta}_{2}^{0}\right)$ are happy to play at or below $r$, and all types above earn nonnegative profits, and are therefore happy to bid a positive amount. And since condition (QM) holds, bidding according to (9) represents their best response. 


\section{APPLICATIONS}

We show how the results developed in the preceding two sections yield results about uniqueness and existence of equilibrium in several bidding games. It must be clear that many more auction games could be examined using the same technology. The following theorem applies to a wide class of games, including almost all the examples we have presented, under the assumption of independent signals. Throughout this section we do not restrict to pure strategy equilibria.

THEOREM 1. Suppose $\theta_{1}, \theta_{2}$ are independent. Then for the games of Examples 1, 2, 4, and 5 satisfying $\mathbf{A 1}$ and $\mathbf{A 3}$, an equilibrium exists and is unique. This equilibrium is in pure strategies and is characterized by (9) and (5).

\section{Proof.}

(1) Regularity of strategies: because of Lemma 4, all equilibrium behavioural strategies are nondecreasing, by Lemma 5 and its corollaries in Appendix A.2 the equilibrium is in nondecreasing pure strategies; by Lemma 6 these strategies are strictly increasing, and differentiable in view of Lemma 7. Appendix A.5 shows that equilibrium trajectories satisfy the Lipschitz condition.

(2) (QM) The general expression for the first-order conditions is equation (8), and we need to check whether increasing $\theta_{i}$ makes this value grow bigger than 0 . In the games of examples $1,2,4$, and $5, \pi_{i}^{L}\left(b, b_{j}, \theta_{i}, \theta_{j}\right)$ is independent of $\theta_{i}$, and $(\partial / \partial b) \pi_{i}^{W}\left(b, b_{j}\left(\theta_{j}\right), \theta_{i}, \theta_{j}\right)$ is nondecreasing in $\theta_{i}$; thus the first-order conditions of expression (8) are increasing (hence, quasimonotone) in $\theta_{i}$.

(3) The North-East envelope is well defined since A7 is verified. The $H_{i}$ functions are strictly quasimonotone in view of Proposition 1, hence the North-East envelope is negatively sloped.

(4) No crossing: since condition (QM) is verified Proposition 4 holds.

Because A7 is verified, a final condition exists and so the discussion in section 3.1 implies that the equilibrium is unique. Moreover, in view of point (1) earlier, it is the unique equilibrium in behavioural strategies.

As for the existence part, all the assumptions of Proposition 5 are met, and therefore an equilibrium in pure strategies exists and is characterized by (9) and (5).

It is interesting to notice that neither the second price auction nor the war of attrition (Example 3) are treated by the above theorem. This is because both fail to meet A7. The second price auction is known to exhibit a continuum of equilibria in the case of common values [see Milgrom (1981); 
Bikchandani and Riley (1991)], although our methods do not apply to that setting. They do apply to the war of attrition, and it turns out that there equilibria display unbounded bidding; so the final condition, whereby the highest types of both players must bid the same amount, fails. In this case we have a continuum of equilibria, each starting from a different point on the North-East envelope. The easiest way to see this is to consider again the benchmark case of independence, considered by Nalebuff and Riley (1985) in the private-values case:

THEOREM 2. In the war of attrition (Example 3) with independent signals there is a continuum of equilibria (in regular strategies).

Proof. In order to prove our claim, we will construct the equilibria. Consider the set of points on the North-East envelope of the curves

$$
H_{i}^{r}\left(\psi_{i}, \psi_{j}\right)=P\left\{\theta_{j} \leq \psi_{j}\right\} E\left(V_{i}\left(\psi_{i}, \theta_{j}\right) \mid \theta_{j} \leq \psi_{j}\right)-r .
$$

Once again these have negative slope, hence the North-East envelope will have the familiar appearance of Figure 2. The expression for the first-order conditions (9) is

$$
\phi_{j}^{\prime}(b) V_{i}\left(\phi_{i}(b), \phi_{j}(b)\right) f_{j}\left(\phi_{j}(b)\right)-\left[1-F_{j}\left(\phi_{j}(b)\right)\right]=0
$$

which is easily seen to satisfy condition (QM); therefore all the points on the North-East envelope are candidate initial conditions and all trajectories starting on the North-East envelope and described by (10) are candidate equilibria. In the other auction forms we disposed of this multiplicity making use of a final condition: No such procedure is allowed here, because any trajectory pair described by equation (10) displays unbounded bidding. To see this, rewrite (10) as follows:

$$
\frac{1}{V_{i}\left(\phi_{i}(b), \phi_{j}(b)\right)}=\phi_{j}^{\prime}(b) \frac{f_{j}\left(\phi_{j}(b)\right)}{1-F_{j}\left(\phi_{j}(b)\right)}=\frac{\partial}{\partial b}\left[-\log \left(1-F_{j}\left(\phi_{j}(b)\right)\right] .\right.
$$

Integrating with respect to $b$ up to $c$ we obtain

$$
\int_{r}^{c} \frac{1}{V_{i}\left(\phi_{i}(s), \phi_{j}(s)\right)} d s=-\log \left(1-F_{j}\left(\phi_{j}(c)\right)+\right.\text { constant. }
$$

Suppose now, by contradiction, that $b_{j}(\cdot)$ were bounded, that is $b_{j}\left(\theta_{j}\right)<$ $K<\infty$. Then necessarily $F_{j}\left(\phi_{j}(K)\right)=1$ whence, evaluating the last expression at $c=K$, the RHS is infinite while the LHS is not; this is the contradiction.

We therefore conclude that all trajectories starting from the North-East envelope and described by equation (10) constitute equilibria for the war of attrition. 
Thus, we see how $\mathbf{A} 7$ is crucial to guarantee uniqueness of equilibrium. Notice that restoring even a minimal amount of "strict decreasingness," like in Examples 4 and 5, suffices to recover uniqueness.

THEOREM 3. Assume that the games of examples 1, 4, and 6 satisfy A1, A2, and A3. Then an equilibrium for these games exists, and is unique in the class of nondecreasing strategy equilibria. This equilibrium is in pure strategies and is characterized by (9) and (5).

Proof. Let us first assume an equilibrium in nondecreasing behavioural strategies exists, and check

(1) Regularity of strategies: because of Lemma 5 and its corollaries in Appendix A.2 the equilibrium is in nondecreasing pure strategies; by Lemma 6 these strategies are strictly increasing, and differentiable in view of Lemma 7. Proposition 6 in Appendix A.5 applies since $H_{i}^{r}$ is quasimonotone [see point (2) below], and shows that equilibrium trajectories satisfy the Lipschitz condition.

(2) (QM) In the case of any auction where the loser pays nothing and the derivative with respect to $b_{i}$ of $\pi_{i}^{W}$ is independent of $b_{j}, \theta_{i}$, and $\theta_{j}$, expression (8) reduces to

$$
\begin{aligned}
& {\left[\phi_{j}^{\prime}(b) \pi_{i}^{W}\left(b, b, \theta_{i}, \phi_{j}(b)\right)+\frac{F_{j}\left(\phi_{j}(b) \mid \theta_{i}\right)}{f_{j}\left(\phi_{j}(b) \mid \theta_{i}\right)} \frac{\partial}{\partial b} \pi_{i}^{W}\left(b, b_{j}\left(\theta_{j}\right), \theta_{i}, \theta_{j}\right)\right]} \\
& \quad \times f_{j}\left(\phi_{j}(b) \mid \theta_{i}\right)=0 .
\end{aligned}
$$

The term in brackets is increasing in $\theta_{i}$ since $\pi_{i}^{W}$ is increasing by $\mathbf{A 6}$, $(\partial / \partial b) \pi_{i}^{W}$ is negative by $\mathbf{A} 7$ and $\left[F_{j}\left(\phi_{j} \mid \theta_{i}\right) / f_{j}\left(\phi_{j} \mid \theta_{i}\right)\right]$ is decreasing in $\theta_{i}$ because of affiliation; this proves quasimonotonicity. This takes care of examples 1 and 4 .

For example 6, expression (8) reduces to

$$
\begin{aligned}
& {\left[\phi_{j}^{\prime}(b) u\left(V\left(\theta_{i}, \phi_{j}(b)\right)-b\right)-\int_{-\infty}^{\phi_{j}(b)} u^{\prime}\left(V\left(\theta_{i}, \phi_{j}(b)\right)-b\right) \frac{f_{j}\left(\theta_{j} \mid \theta_{i}\right)}{f_{j}\left(\phi_{j}(b) \mid \theta_{i}\right)} d \theta_{j}\right]} \\
& \quad \times f_{j}\left(\phi_{j}(b) \mid \theta_{i}\right)=0 .
\end{aligned}
$$

The term in brackets is strictly increasing in $\theta_{i}$, since $u$ is concave and $\left[f_{j}\left(\theta_{j} \mid \theta_{i}\right) / f_{j}\left(\phi_{j}(b) \mid \theta_{i}\right)\right]$ is nonincreasing in $\theta_{i}$ for $\theta_{j}<\phi_{j}$ due to affiliation. This yields quasimonotonicity of the first-order conditions.

(3) The North-East envelope is well defined since A7 is verified. The $H_{i}$ are strictly quasimonotone in view of Proposition 1 (observe that in this case $\mathbf{A 8}$ is verified, in view of the discussion preceding Proposition 1), and thus the North-East envelope is negatively sloped. 
(4) No crossing: condition $(\mathbf{Q M})$ is verified and therefore Proposition 4 holds.

Because A7 is verified, a final condition exists and so the discussion in section 3.1 implies that the equilibrium is unique. Moreover, in view of point (1) earlier, it is the unique equilibrium in nondecreasing behavioural strategies.

As for the existence part, all the assumptions of Proposition 5 are met, and therefore an equilibrium in pure strategies exists and is characterized by (9) and (5).

In auctions where the loser pays, affiliation is not a sufficient condition for the existence of equilibria in nondecreasing strategies because affiliation does not guarantee that condition $\mathbf{Q M}$ is satisfied in such games. The following assumption is imposed to guarantee that in these games condition QM holds and that therefore we get existence of equilibria in nondecreasing strategies. The assumption reduces to the assumptions made in Krishna-Morgan (1997) for the war of attrition and the all-pay auction. It is clearly satisfied if the signals are independent.

A9. The expression $K^{\alpha}\left(\theta_{i}, \theta_{j}\right):=V_{i}\left(\theta_{i}, \theta_{j}\right)\left[f_{j}\left(\theta_{j} \mid \theta_{i}\right) / 1-(1-\alpha) F_{j}\right.$. $\left.\left(\theta_{j} \mid \theta_{i}\right)\right]$ is nondecreasing in $\theta_{i}$.

THEOREM 4. Assume that the games of Examples 2 and 5 satisfy A1, A2, $\mathbf{A 3}$, and A9. Then an equilibrium for these games exists and is unique in the class of nondecreasing strategy equilibria. This equilibrium is in pure strategies and is characterized by (9) and (5).

Proof. The proof is the same as the one of Theorem 3 except for:

- Point (3) of the above proof: here we make reference to Proposition 2 to establish that the North-East envelope is well defined and negatively sloped. To see that the assumptions of that proposition are verified, observe that, if $K^{\alpha}\left(\theta_{i}, \theta_{j}\right)$ is increasing in $\theta_{i}$, then $V_{i}\left(\theta_{i}, \theta_{j}\right) f_{j}\left(\theta_{j} \mid \theta_{i}\right)$ is increasing in $\theta_{i}$.

- Verifying (QM): In the all-pay auction, expression (8) simplifies to

$$
\phi_{j}^{\prime}(b) V\left(\theta_{i}, \phi_{j}(b)\right) f_{j}\left(\phi_{j}(b) \mid \theta_{i}\right)-1=0,
$$

which is quasimonotone (indeed, increasing) in $\theta_{i}$ if $V\left(\theta_{i}, \phi_{j}\right) f_{j}\left(\phi_{j} \mid \theta_{i}\right)$ is increasing in $\theta_{i}$. It is possible to see that, for a mechanism where winner and loser pay the same combination of the winner's and loser's bid, the same condition guarantees an equilibrium in increasing strategies. In the war of attrition, expression (8) simplifies to

$$
\left[\phi_{j}^{\prime}(b) V\left(\theta_{i}, \phi_{j}(b)\right) \frac{f_{j}\left(\phi_{j}(b) \mid \theta_{i}\right)}{1-F_{j}\left(\phi_{j}(b) \mid \theta_{i}\right)}-1\right]\left(1-F_{j}\left(\phi_{j}(b) \mid \theta_{i}\right)\right)=0,
$$


which is quasimonotone if $V\left(\theta_{i}, \phi_{j}\right)\left[f_{j}\left(\phi_{j} \mid \theta_{i}\right) /\left(1-F_{j}\left(\phi_{j} \mid \theta_{i}\right)\right)\right]$ is increasing in $\theta_{i}$. Of these two conditions, the latter is more demanding than the former, and they have been proposed by Krishna and Morgan in (1997). For the combination of war of attrition and all-pay auction presented in Example 5, the relevant condition is that $V\left(\theta_{i}, \phi_{j}\right)\left[f_{j}\left(\phi_{j} \mid \theta_{i}\right) /\right.$ $\left.\left(1-(1-\alpha) F_{j}\left(\phi_{j} \mid \theta_{i}\right)\right)\right]$ be increasing in $\theta_{i}$.

\section{APPENDIX: REGULARITY OF EQUILIBRIUM STRATEGIES}

This section provides sufficient conditions for regularity of equilibrium strategies. The assumptions required are fairly general. Reserve prices are not necessary except for the proof of the Lipschitz condition in Proposition 6 .

The structure of this section is sequential, in that an increasing level of regularity is proved. Except for the case of independence we only discuss the class of equilibria in nondecreasing behavioural strategies.

\section{A.1. Independence}

Here we prove that, if signals are independent, all equilibria in behavioural strategies are nondecreasing. Together with the subsequent results, this will yield a complete characterization of the equilibrium set for this case. We assume that the payoff functions $\pi_{i}\left(b_{i}, b_{j}, \theta_{i}, \theta_{j}\right)$ are additively separable in $b_{i}$ and $\theta_{i}$.

A10. $\pi_{i}\left(b_{i}, b_{j}, \theta_{i}, \theta_{j}\right)=\rho_{i}\left(b_{i}, b_{j}, \theta_{j}\right)+\sigma_{i}\left(b_{j}, \theta_{i}, \theta_{j}\right)$.

Lemma 4. Assume A1, A3, A6, A7, and A10. Suppose $\Theta_{i}, \Theta_{j}$ are independent. Then equilibrium behavioural strategies are nondecreasing.

Proof. We will prove that, if some type $\theta_{i}$ wins the object with some (nonzero) probability, then all types greater than $\theta_{i}$ will bid at least as much. By contradiction, suppose $b_{i} \geq r$, and

$$
\theta_{i}^{\prime}>\theta_{i} \text { but } \operatorname{support}\left(\eta_{i}\left(\cdot, \theta_{i}^{\prime}\right)\right) \ni b^{\prime}<b \in \operatorname{support}\left(\eta_{i}\left(\cdot, \theta_{i}\right)\right) .
$$

Optimality of the bidding function $\eta_{i}(\cdot)$ implies

$$
\Pi\left(\theta_{i}, b, \eta_{j}\right) \geq \Pi\left(\theta_{i}, b^{\prime}, \eta_{j}\right)
$$

and

$$
\Pi\left(\theta_{i}^{\prime}, b^{\prime}, \eta_{j}\right) \geq \Pi\left(\theta_{i}^{\prime}, b, \eta_{j}\right) .
$$


Switching the LHS with the RHS in the first inequality, and adding the second one gives

$$
\Pi\left(\theta_{i}^{\prime}, b^{\prime}, \eta_{j}\right)-\Pi\left(\theta_{i}, b^{\prime}, \eta_{j}\right) \geq \Pi\left(\theta_{i}^{\prime}, b, \eta_{j}\right)-\Pi\left(\theta_{i}, b, \eta_{j}\right) .
$$

This inequality may be rewritten as

$$
\begin{gathered}
\int_{\left(-\infty, b^{\prime}\right) \times[\underline{\theta}, \bar{\theta}]}\left[\pi_{i}^{W}\left(b^{\prime}, s \vee r, \theta_{i}^{\prime}, \theta_{j}\right)-\pi_{i}^{W}\left(b^{\prime}, s \vee r, \theta_{i}, \theta_{j}\right)\right] \mu_{j}\left(d s, d \theta_{j}\right) \\
+\int_{\left(b^{\prime},+\infty\right) \times[\underline{\theta}, \bar{\theta}]}\left[\pi_{i}^{L}\left(b^{\prime}, s, \theta_{i}^{\prime}, \theta_{j}\right)-\pi_{i}^{L}\left(b^{\prime}, s, \theta_{i}, \theta_{j}\right)\right] \mu_{j}\left(d s, d \theta_{j}\right) \\
+\frac{1}{2} \int_{\left\{b^{\prime}\right\} \times[\underline{\theta}, \bar{\theta}]}\left[\pi_{i}^{W}\left(b^{\prime}, s \vee r, \theta_{i}^{\prime}, \theta_{j}\right)-\pi_{i}^{W}\left(b^{\prime}, s \vee r, \theta_{i}, \theta_{j}\right)\right. \\
\left.\quad+\pi_{i}^{L}\left(b^{\prime}, s, \theta_{i}^{\prime}, \theta_{j}\right)-\pi_{i}^{L}\left(b^{\prime}, s, \theta_{i}, \theta_{j}\right)\right] \mu_{j}\left(d s, d \theta_{j}\right) \\
\geq \int_{(-\infty, b) \times[\underline{\theta}, \bar{\theta}]}\left[\pi_{i}^{W}\left(b, s \vee r, \theta_{i}^{\prime}, \theta_{j}\right)-\pi_{i}^{W}\left(b, s \vee r, \theta_{i}, \theta_{j}\right)\right] \mu_{j}\left(d s, d \theta_{j}\right) \\
\quad+\int_{(b,+\infty) \times[\underline{\theta}, \bar{\theta}]}\left[\pi_{i}^{L}\left(b, s, \theta_{i}^{\prime}, \theta_{j}\right)-\pi_{i}^{L}\left(b, s, \theta_{i}, \theta_{j}\right)\right] \mu_{j}\left(d s, d \theta_{j}\right) \\
\quad+\frac{1}{2} \int_{\{b\} \times[\underline{\theta}, \bar{\theta}]}\left[\pi_{i}^{W}\left(b, s \vee r, \theta_{i}^{\prime}, \theta_{j}\right)-\pi_{i}^{W}\left(b, s \vee r, \theta_{i}, \theta_{j}\right)\right. \\
\left.+\pi_{i}^{L}\left(b, s, \theta_{i}^{\prime}, \theta_{j}\right)-\pi_{i}^{L}\left(b, s, \theta_{i}, \theta_{j}\right)\right] \mu_{j}\left(d s, d \theta_{j}\right) .
\end{gathered}
$$

Because of the separability assumption, the arguments inside the integrals do not depend on type $i$ 's bid, and recalling that $b^{\prime}<b$, the above is equivalent to

$$
\begin{aligned}
& 0 \geq \int_{\left(b^{\prime}, b\right) \times[\underline{\theta}, \bar{\theta}]}\left[\pi_{i}^{W}\left(b, s \vee r, \theta_{i}^{\prime}, \theta_{j}\right)-\pi_{i}^{W}\left(b, s \vee r, \theta_{i}, \theta_{j}\right)\right] \mu_{j}\left(d s, d \theta_{j}\right) \\
& \quad+\int_{\left(b^{\prime}, b\right) \times[\underline{\theta}, \bar{\theta}]}\left[\pi_{i}^{L}\left(b, s, \theta_{i}^{\prime}, \theta_{j}\right)-\pi_{i}^{L}\left(b, s, \theta_{i}, \theta_{j}\right)\right] \mu_{j}\left(d s, d \theta_{j}\right) \\
& +\frac{1}{2} \int_{\{b\} \times[\underline{\theta}, \bar{\theta}]}\left[\pi_{i}^{W}\left(b, s \vee r, \theta_{i}^{\prime}, \theta_{j}\right)-\pi_{i}^{W}\left(b, s \vee r, \theta_{i}, \theta_{j}\right)\right. \\
& \left.\quad+\pi_{i}^{L}\left(b, s, \theta_{i}^{\prime}, \theta_{j}\right)-\pi_{i}^{L}\left(b, s, \theta_{i}, \theta_{j}\right)\right] \mu_{j}\left(d s, d \theta_{j}\right) \\
& +\frac{1}{2} \int_{\left\{b^{\prime}\right\} \times[\underline{\theta}, \bar{\theta}]}\left[\pi_{i}^{W}\left(b, s \vee r, \theta_{i}^{\prime}, \theta_{j}\right)-\pi_{i}^{W}\left(b, s \vee r, \theta_{i}, \theta_{j}\right)\right. \\
& \left.\quad+\pi_{i}^{L}\left(b, s, \theta_{i}^{\prime}, \theta_{j}\right)-\pi_{i}^{L}\left(b, s, \theta_{i}, \theta_{j}\right)\right] \mu_{j}\left(d s, d \theta_{j}\right) .
\end{aligned}
$$

But by $\mathbf{A 6}$ the arguments of the integrals are strictly positive. To reach a contradiction, it is enough to prove that at least one the supports of integration on the RHS has nonzero $\mu_{j}$ measure. This follows from the 
fact that, were it not so, type $\theta_{i}$ would strictly prefer to lower his bid to $b^{\prime}$ (A7), winning with the same probability (which is nonzero by A3) and lowering his payment. Thus, the RHS is strictly positive.

\section{A.2. Continuity}

This lemma and the following corollaries show that an equilibrium in nondecreasing behavioural strategies is in pure strategies that are continuous and nondecreasing.

Lemma 5. Assume A1, A3, and A7. Consider any open interval $(\alpha, \beta) \subset$ $(r, B) .{ }^{3}$ Then, in a nondecreasing strategies equilibrium, $\mu_{j}((\alpha, \beta))>0 \forall j$.

Proof. Suppose not. Then there is an open interval $(\alpha, \beta)$ such that either

(I) $\mu_{i}((\alpha, \beta))>0$ but $\mu_{j}((\alpha, \beta))=0$ for some $i \neq j$.

or

(II) $\mu_{i}((\alpha, \beta))=0$ for all $i$.

Case (I): Let $b \in(\alpha, \beta)$ be a bid that is played by some $\theta_{i}$; then player $\theta_{i}$ prefers bidding $(b+\alpha) / 2$ to bidding $b$. The reason for this can be easily seen: The payoff to this player is:

$$
\begin{aligned}
& \int_{(-\infty, b) \times[\underline{\theta}, \bar{\theta}]} \pi_{i}^{W}\left(b, s \vee r, \theta_{i}, \theta_{j}\right) d \mu_{j}\left(d s, d \theta_{j} \mid \theta_{i}\right) \\
& \quad+\int_{(b, \infty) \times[\underline{\theta}, \bar{\theta}]} \pi_{i}^{L}\left(b, s \vee r, \theta_{i}, \theta_{j}\right) d \mu_{j}\left(d s, d \theta_{j} \mid \theta_{i}\right) .
\end{aligned}
$$

By lowering $b$ to $(b+\alpha) / 2$ he only changes the limits of integration on a set of zero $\mu_{j}$ measure and by assumption A7 and $\mathbf{A 3}$, the integrand of the first term strictly increases and that of the second does not fall. Contradiction.

Case (II): Let $(\gamma, \delta)$ be the largest open interval containing $(\alpha, \beta)$ such that $\mu_{i}(\gamma, \delta)=0 \forall i$. We must distinguish three subcases:

(i) $\mu_{i}(\{\delta\})>0$ and $\mu_{j}(\{\delta\})=0$, i.e. there is a mass at $\delta$ in the distribution of player $i$ 's bids.

If this is the case, it must be true that there exists a positive measure of types of player $i$ bidding $\delta$ with positive probability. But any such type will prefer to bid $(\delta+\gamma) / 2$ for the same reason as earlier. Contradiction.

(ii) $\mu_{1}(\{\delta\}) \vee \mu_{2}(\{\delta\})=0$.

\footnotetext{
${ }^{3}$ Recall that $B$ was defined as the supremum of all bids ever played in equilibrium.
} 
By definition of $\delta$, for all $\zeta>\delta$ for both $i$ 's, there is a positive mass of types bidding in $(\zeta, \delta)$. However, picking $\zeta$ arbitrarily close to $\delta$ we can make this mass arbitrarily small. Hence, any such type that bids $(\gamma+\delta) / 2$ instead changes the limits of integration in (11) by a negligible mass but increases the integrands by a nonnegligible amount. Contradiction.

(iii) $\mu_{1}(\{\delta\}) \wedge \mu_{2}(\{\delta\})>0$, i.e. both players have a mass at $\delta$.

This cannot happen. To see this, notice that the payoff from bidding $\delta$ minus the payoff from bidding $\delta-\epsilon$ converges as $\epsilon$ converges to zero, to

$$
\frac{1}{2} \int_{\{\delta\} \times[\underline{\theta}, \bar{\theta}]}\left(\pi_{i}^{W}\left(\delta, \delta, \theta_{i}, \theta_{j}\right)-\pi_{i}^{L}\left(\delta, \delta, \theta_{i}, \theta_{j}\right)\right) d \mu_{j}
$$

The payoff from bidding $\delta+\epsilon$ minus the payoff from bidding $\delta$ also converges to the above expression as $\epsilon$ converges to zero. Since types who bid $\delta$ do not wish to bid $\delta+\epsilon$ for any $\epsilon$, the increase in payoff from bidding $\delta$ instead of $\delta-\epsilon$ is infinitesimal. But the increase in payoff from bidding $(\gamma+\delta) / 2$ instead of $\delta-\epsilon$ is nonnegligible. Thus, it is better to bid $(\gamma+\delta) / 2$ instead of $\delta$. Contradiction.

We have exhausted all possible cases, and we have therefore proved the lemma.

Remarks. 1. The above Lemma extends to all intervals with positive Lebesgue measure, observing that any such interval contains an open interval.

2. Lemma 5 implies, in particular, $B_{i}=B_{j}$.

3. Lemma 5 implies that, in equilibrium, bidding starts at, or just above, $r{ }^{4}$

4. The war of attrition does not satisfy assumption A7. However it is easy to see that Lemma 5 would still hold if we assumed that $\pi^{L}$ is strictly decreasing in $b$; so the war of attrition also satisfies the above Lemma.

Corollary 1. Assume A1, A3, and A7. Then an equilibrium in nondecreasing strategies must be in pure strategies.

Proof. By the definition of nondecreasing strategies, if there was one type of a player playing a nonpure strategy, then all types above and below him could not include the interior of the support of that type's strategy in their support. Thus, there would be a set of bids which is played with 0 probability, contradicting Lemma 5.

\footnotetext{
${ }^{4}$ This is not true for every bidding game, for example, in the symmetric equilibrium of the second price auction there is a gap between $r$ and the first active bids. The second price auction does not satisfy assumption A 7 .
} 
We define $b_{i}^{-1}(\cdot)$ to be the (set-valued) inverse correspondence associated to $b_{i}(\cdot)$.

Corollary 2. Assume A1, A3, A7, and that equilibrium strategies are nondecreasing. Then equilibrium strategies $b_{i}(\cdot)$ are continuous on $b_{i}^{-1}(r, B]$.

Proof. Observe that, by Corollary 1, we can restrict attention to pure strategies. Then the statement follows immediately from the assumption that strategies are nondecreasing, coupled with Lemma 5.

\section{A.3. Strictly Increasing}

This section gives conditions under which a nondecreasing pure strategy is strictly increasing for the types that bid above $r$. An implication of this is that it cannot be that a mass of types of any bidder will ever bid at any amount $b>r$. To this end we need to introduce an assumption that is satisfied in all our examples.

A11. $\pi_{i}^{L}\left(b_{i}, b_{j}, \theta_{i}, \theta_{j}\right)$ is nonincreasing in $\theta_{j}$.

Lemma 6. Assume A1, A3, A4, A6, A7, A11, and that equilibrium strategies $b_{i}(\cdot)$ are nondecreasing. Then equilibrium strategies are strictly increasing on $\left(b_{i}^{-1}(r), \bar{\theta}\right]$.

Proof. Suppose not. Then there is a $b \in(r, B]$ such that $P\left\{b_{j}\left(\theta_{j}\right)=b \mid\right.$ $\left.\theta_{i}\right\}>0$ for every $\theta_{i}$. If this is the case, we will show that no type of player $i$ will want to bid in $(b-\epsilon, b)$ for some small $\epsilon$, and this will contradict Lemma 5.

In view of Corollary 1 above, equilibrium strategies are pure; hence the payoff to some $\theta_{i, \epsilon}$ who bids $b-\epsilon$ is:

$$
\begin{aligned}
\Pi(b-\epsilon & \left., \theta_{i, \epsilon}, b_{j}(\cdot)\right) \\
= & \int_{\left[\theta_{j}:\left(b-\epsilon>b_{j}\left(\theta_{j}\right)\right)\right]} \pi_{i}^{W}\left(b-\epsilon, b_{j}\left(\theta_{j}\right), \theta_{i, \epsilon}, \theta_{j}\right) d f_{j}\left(\theta_{j} \mid \theta_{i, \epsilon}\right) \\
& +\int_{\left[\theta_{j}:\left(b-\epsilon<b_{j}\left(\theta_{j}\right)\right]\right.} \pi_{i}^{L}\left(b-\epsilon, b_{j}\left(\theta_{j}\right), \theta_{i, \epsilon}, \theta_{j}\right) d f_{j}\left(\theta_{j} \mid \theta_{i, \epsilon}\right) \\
& +\frac{1}{2} \int_{\left[\theta_{j}: b-\epsilon=b_{j}\left(\theta_{j}\right)\right]}\left[\pi_{i}^{W}\left(b-\epsilon, b_{j}\left(\theta_{j}\right), \theta_{i, \epsilon}, \theta_{j}\right)\right. \\
& \left.+\pi_{i}^{L}\left(b-\epsilon, b_{j}\left(\theta_{j}\right), \theta_{i, \epsilon}, \theta_{j}\right)\right] d f_{j}\left(\theta_{j} \mid \theta_{i, \epsilon}\right) .
\end{aligned}
$$

As $\epsilon$ goes to zero, $b-\epsilon$ converges to $b-$ and $\theta_{i, \epsilon}$ to some $\theta_{i}$, and the payoff converges to:

$$
\begin{aligned}
\Pi\left(b-, \theta_{i}, b_{j}(\cdot)\right)= & \int_{\left[\theta_{j}:\left(b->b_{j}\left(\theta_{j}\right)\right)\right]} \pi_{i}^{W}\left(b, b_{j}\left(\theta_{j}\right), \theta_{i}, \theta_{j}\right) d f_{j}\left(\theta_{j} \mid \theta_{i}\right), \\
& +\int_{\left[\theta_{j}:\left(b-<b_{j}\left(\theta_{j}\right)\right]\right.} \pi_{i}^{L}\left(b, b_{j}\left(\theta_{j}\right), \theta_{i}, \theta_{j}\right) d f_{j}\left(\theta_{j} \mid \theta_{i}\right),
\end{aligned}
$$


where we have replaced $b-\epsilon$ with $b$ inside the integrals (although not in the supports), since by $\mathbf{A} 4$ the payoff is continuous. Since all types $\theta_{i, \epsilon}$ were happy to bid $b-\epsilon$, and did not want to decrease their bid to $b-2 \epsilon$, taking the limit it has to be true that

$$
\pi_{i}^{W}\left(b, b, \theta_{i}, b_{j}^{-1}(b-)\right)-\pi_{i}^{L}\left(b, b, \theta_{i}, b_{j}^{-1}(b-)\right) \geq 0 .
$$

Observe now that type $\theta_{i}-\epsilon$, who by construction bids less than $b$, as $\epsilon$ goes to 0 should prefer to bid slightly above $b$. Indeed he would be gaining

$$
\begin{aligned}
& \Pi\left(b+, \theta_{i}, b_{j}(\cdot)\right)-\Pi\left(b-, \theta_{i}, b_{j}(\cdot)\right) \\
& \quad=\int_{b_{j}^{-1}(b-)}^{b_{j}^{-1}(b+)}\left[\pi_{i}^{W}\left(b, b, \theta_{i}, \theta_{j}\right)-\pi_{i}^{L}\left(b, b, \theta_{i}, \theta_{j}\right)\right] d f_{j}\left(\theta_{j} \mid \theta_{i}\right),
\end{aligned}
$$

and the above expression is just the integral of (12), as $\theta_{j}$ increases from $b_{j}^{-1}(b-)$ upwards. But the integrand of (13) is nonnegative at the lower bound of the integral by (12), and is strictly increasing in $\theta_{j}$ by $\mathbf{A 6}$ and A11; thus, the integrand in expression (13) is positive over the domain of integration, and expression (13) is positive too. So type $\theta_{i}-\epsilon$ will never want to bid $b-\epsilon$ for $\epsilon$ small enough, and this contradicts Lemma 5.

\section{A.4. Differentiability}

Lemma 7. Assume A1, A3, A4, A6, A7, A11, and that equilibrium strategies $b_{i}(\cdot)$ are strictly increasing. Then equilibrium inverse bidding functions are everywhere differentiable in the interior of their domain.

Proof. Abusing notation, let $\pi^{W}\left(b_{i}, b_{j}\left(\theta_{j}\right), \theta_{i}, \theta_{j}\right)$ be denoted by $\pi^{W}\left(b_{i}\right.$, $\left.\theta_{i}, \theta_{j}\right)$, and similarly for $\pi^{L}$.

Fix any $\theta_{i} \in\left(\theta_{i}^{0}, \overline{\theta_{i}}\right)$, consider any increasing sequence $\left\{\theta_{i}^{n}\right\} \uparrow \theta_{i}$; let $b_{i}^{n}:=b_{i}\left(\theta_{i}^{n}\right), b_{i}:=b_{i}\left(\theta_{i}\right)$. Continuity of $b_{i}(\cdot)$ (Corollary 2) implies $b_{i}^{n} \uparrow b_{i}$.

Because types $\theta_{i}^{n}$ prefer to bid $b_{i}^{n}$ rather than $b_{i}$, we can write

$$
\begin{aligned}
\int_{\underline{\theta}}^{\phi_{j}\left(b_{i}^{n}\right)} & \pi^{W}\left(b_{i}^{n}, \theta_{i}^{n}, \theta_{j}\right) f_{j}\left(\theta_{j} \mid \theta_{i}^{n}\right) d \theta_{j}+\int_{\phi_{j}\left(b_{i}^{n}\right)}^{\bar{\theta}} \pi^{L}\left(b_{i}^{n}, \theta_{i}^{n}, \theta_{j}\right) f_{j}\left(\theta_{j} \mid \theta_{i}^{n}\right) d \theta_{j} \\
\geq & \int_{\underline{\theta}}^{\phi_{j}\left(b_{i}\right)} \pi^{W}\left(b_{i}, \theta_{i}^{n}, \theta_{j}\right) f_{j}\left(\theta_{j} \mid \theta_{i}^{n}\right) d \theta_{j} \\
& +\int_{\phi_{j}\left(b_{i}\right)}^{\bar{\theta}} \pi^{L}\left(b_{i}, \theta_{i}^{n}, \theta_{j}\right) f_{j}\left(\theta_{j} \mid \theta_{i}^{n}\right) d \theta_{j} .
\end{aligned}
$$

Subtracting from both sides the expression

$$
\int_{\underline{\theta}}^{\phi_{j}\left(b_{i}^{n}\right)} \pi^{W}\left(b_{i}, \theta_{i}^{n}, \theta_{j}\right) f_{j}\left(\theta_{j} \mid \theta_{i}^{n}\right) d \theta_{j}+\int_{\phi_{j}\left(b_{i}^{n}\right)}^{\bar{\theta}} \pi^{L}\left(b_{i}, \theta_{i}^{n}, \theta_{j}\right) f_{j}\left(\theta_{j} \mid \theta_{i}^{n}\right) d \theta_{j},
$$


we obtain

$$
\begin{gathered}
\int_{\underline{\theta}}^{\phi_{j}\left(b_{i}^{n}\right)}\left[\pi^{W}\left(b_{i}^{n}, \theta_{i}^{n}, \theta_{j}\right)-\pi^{W}\left(b_{i}, \theta_{i}^{n}, \theta_{j}\right)\right] f_{j}\left(\theta_{j} \mid \theta_{i}^{n}\right) d \theta_{j} \\
+\int_{\phi_{j}\left(b_{i}^{n}\right)}^{\bar{\theta}}\left[\pi^{L}\left(b_{i}^{n}, \theta_{i}^{n}, \theta_{j}\right)-\pi^{L}\left(b_{i}, \theta_{i}^{n}, \theta_{j}\right)\right] f_{j}\left(\theta_{j} \mid \theta_{i}^{n}\right) d \theta_{j} \\
\geq \int_{\phi_{j}\left(b_{i}^{n}\right)}^{\phi_{j}\left(b_{i}\right)} \pi^{W}\left(b_{i}, \theta_{i}^{n}, \theta_{j}\right) f_{j}\left(\theta_{j} \mid \theta_{i}^{n}\right) d \theta_{j} \\
\quad-\int_{\phi_{j}\left(b_{i}^{n}\right)}^{\phi_{j}\left(b_{i}\right)} \pi^{L}\left(b_{i}, \theta_{i}^{n}, \theta_{j}\right) f_{j}\left(\theta_{j} \mid \theta_{i}^{n}\right) d \theta_{j} .
\end{gathered}
$$

Dividing both sides by $b_{i}-b_{i}^{n}$, and taking limsup yields

$$
\begin{aligned}
& \int_{\underline{\theta}}^{\phi_{j}\left(b_{i}\right)}-\frac{\partial}{\partial b_{i}} \pi^{W}\left(b_{i}, \theta_{i}, \theta_{j}\right) f_{j}\left(\theta_{j} \mid \theta_{i}\right) d \theta_{j} \\
& +\int_{\phi_{j}\left(b_{i}\right)}^{\bar{\theta}}-\frac{\partial}{\partial b_{i}} \pi^{L}\left(b_{i}, \theta_{i}, \theta_{j}\right) f_{j}\left(\theta_{j} \mid \theta_{i}\right) d \theta_{j} \\
& \geq \limsup _{n \rightarrow \infty} \int_{\phi_{j}\left(b_{i}^{n}\right)}^{\phi_{j}\left(b_{i}\right)}\left[\pi^{W}\left(b_{i}, \theta_{i}^{n}, \theta_{j}\right)-\pi^{L}\left(b_{i}, \theta_{i}^{n}, \theta_{j}\right)\right] \\
& \quad \times f_{j}\left(\theta_{j} \mid \theta_{i}^{n}\right) d \theta_{j} \frac{1}{b_{i}-b_{i}^{n}} .
\end{aligned}
$$

The RHS, by continuity of $\pi^{W}-\pi^{L}$ in $\theta_{i}, \theta_{j}$, can be replaced by

$$
\begin{aligned}
& {\left[\pi^{W}\left(b_{i}, \theta_{i}, \phi_{j}\left(b_{i}\right)\right)-\pi^{L}\left(b_{i}, \theta_{i}, \phi_{j}\left(b_{i}\right)\right)\right] f_{j}\left(\phi_{j}\left(b_{i}\right) \mid \theta_{i}\right)} \\
& \quad \times \limsup _{n \rightarrow \infty} \int_{\phi_{j}\left(b_{i}^{n}\right)}^{\phi_{j}\left(b_{i}\right)} d \theta_{j} \frac{1}{b_{i}-b_{i}^{n}} .
\end{aligned}
$$

Therefore,

$$
\begin{aligned}
\limsup _{b_{i}^{n} \uparrow b_{i}} & \frac{\phi_{j}\left(b_{i}\right)-\phi_{j}\left(b_{i}^{n}\right)}{b_{i}-b_{i}^{n}} \\
& \leq \frac{\int_{\underline{\theta}}^{\phi_{j}\left(b_{i}\right)}-\frac{\partial}{\partial b_{i}} \pi^{W}\left(b_{i}, \theta_{i}, \theta_{j}\right) f_{j}\left(\theta_{j} \mid \theta_{i}\right) d \theta_{j}+\int_{\phi_{j}\left(b_{i}\right)}^{\bar{\theta}}-\frac{\partial}{\partial b_{i}} \pi^{L}\left(b_{i}, \theta_{i}, \theta_{j}\right) f_{j}\left(\theta_{j} \mid \theta_{i}\right) d \theta_{j}}{\left[\pi^{W}\left(b_{i}, \theta_{i}, \phi_{j}\left(b_{i}\right)\right)-\pi^{L}\left(b_{i}, \theta_{i}, \phi_{j}\left(b_{i}\right)\right)\right] f_{j}\left(\phi_{j}\left(b_{i}\right) \mid \theta_{i}\right)} .
\end{aligned}
$$

A symmetric reasoning leads to

$$
\begin{aligned}
\liminf _{b_{i}^{n} \dagger b_{i}} & \frac{\phi_{j}\left(b_{i}\right)-\phi_{j}\left(b_{i}^{n}\right)}{b_{i}-b_{i}^{n}} \\
& \geq \frac{\int_{\underline{\theta}}^{\phi_{j}\left(b_{i}\right)}-\frac{\partial}{\partial b_{i}} \pi^{W}\left(b_{i}, \theta_{i}, \theta_{j}\right) f_{j}\left(\theta_{j} \mid \theta_{i}\right) d \theta_{j}+\int_{\phi_{j}\left(b_{i}\right)}^{\bar{\theta}}-\frac{\partial}{\partial b_{i}} \pi^{L}\left(b_{i}, \theta_{i}, \theta_{j}\right) f_{j}\left(\theta_{j} \mid \theta_{i}\right) d \theta_{j}}{\left[\pi^{W}\left(b_{i}, \theta_{i}, \phi_{j}\left(b_{i}\right)\right)-\pi^{L}\left(b_{i}, \theta_{i}, \phi_{j}\left(b_{i}\right)\right)\right] f_{j}\left(\phi_{j}\left(b_{i}\right) \mid \theta_{i}\right)} .
\end{aligned}
$$


Because the same exercise can be carried out choosing a decreasing sequence $\left\{\theta_{i}^{n}\right\} \downarrow \theta_{i}$, we conclude that $\phi_{j}(\cdot)$ is differentiable everywhere inside its domain.

\section{A.5. Lipschitz Condition}

Equilibrium trajectories are described by the following system of firstorder conditions

$\phi_{j}^{\prime}(b)$

$$
=\frac{\int_{\underline{\theta}}^{\phi_{j}(b)}-\frac{\partial}{\partial b} \pi_{i}^{W}\left(b, b_{j}\left(\theta_{j}\right), \phi_{i}, \theta_{j}\right) f_{j}\left(\theta_{j} \mid \phi_{i}\right) d \theta_{j}+\int_{\phi_{j}(b)}^{\bar{\theta}}-\frac{\partial}{\partial b} \pi_{i}^{L}\left(b, b_{j}\left(\theta_{j}\right), \phi_{i}, \theta_{j}\right) f_{j}\left(\theta_{j} \mid \phi_{i}\right) d \theta_{j}}{\left\{\pi_{i}^{W}\left(b, b, \phi_{i}, \phi_{j}(b)\right)-\pi_{i}^{L}\left(b, b, \phi_{i}, \phi_{j}(b)\right)\right\} f_{j}\left(\phi_{j}(b) \mid \phi_{i}\right)}
$$

In order to show that the trajectories described by (14) are Lipschitz continuous, we need to show that the denominator of (14) is bounded away from 0. When A1 holds, it suffices to check that the terms in brackets is bounded away from 0 . Thus, trajectories in the war of attrition and all-pay auctions are easily seen to be Lipschitz. Furthermore, all auctions where the price paid when winning and losing are linear (though not necessarily convex) combinations of the winner's and loser's bid have Lipschitz trajectories for the same reason, provided that the value of the object is strictly positive.

The next assumption imposes that one would prefer the opponent to bid low when one is winning but to bid high when one is losing. It is satisfied by the war of attrition, all-pay auction and combinations of the two because for these games $\pi_{i}^{L}$ is independent of $b_{j}$. It is clearly satisfied by combinations of first price and second-price auctions.

A12. $\pi_{i}^{W}\left(b_{i}, b_{j}, \theta_{i}, \theta_{j}\right)$ is nonincreasing in $b_{j}$ and $\pi_{i}^{L}\left(b_{i}, b_{j}, \theta_{i}, \theta_{j}\right)$ is nondecreasing in $b_{j}$.

Proposition 6. Assume A1, A6, A11, A5, A12, and that condition (QM) holds. Suppose further that $H_{i}^{r}\left(\psi_{i}, \psi_{j}\right)$ is quasimonotone in $\psi_{i}$. Then the system of equilibrium trajectories described by (14) is Lipschitz on $\left(r, \bar{b}\left(\theta_{1}^{0}, \theta_{2}^{0}\right)\right]$.

Proof. We need to prove that $\left[\pi_{i}^{W}\left(b, b, \phi_{i}, \phi_{j}(b)\right)-\pi_{i}^{L}\left(b, b, \phi_{i}\right.\right.$, $\left.\left.\phi_{j}(b)\right)\right]>k>0$. So, let us write the following chain of inequalities

$P\left(\theta_{j} \leq \phi_{j}(b) \mid \theta_{i}=\phi_{i}\right)\left[\pi_{i}^{W}\left(b, b, \phi_{i}, \phi_{j}(b)\right)-\pi_{i}^{L}\left(b, b, \phi_{i}, \phi_{j}(b)\right)+\epsilon\right]$

[since $\pi_{i}^{W}\left(b, b, \phi_{i}, \phi_{j}(b)\right)-\pi_{i}^{L}\left(b, b, \phi_{i}, \phi_{j}(b)\right)$ is increasing in $\theta_{j}$ by $\mathbf{A 6}$ ],

$$
\begin{aligned}
> & P\left(\theta_{j} \leq \phi_{j}(b) \mid \theta_{i}=\phi_{i}\right) \\
& \times E\left(\pi_{i}^{W}\left(b, b, \theta_{i}, \theta_{j}\right)-\pi_{i}^{L}\left(b, b, \theta_{i}, \theta_{j}\right) \mid \theta_{i}=\phi_{i}, \theta_{j} \leq \phi_{j}(b)\right)
\end{aligned}
$$


[since we are adding a nonpositive quantity by $\mathbf{A 5}$ ],

$\geq P\left(\theta_{j} \leq \phi_{j}(b) \mid \theta_{i}=\phi_{i}\right)$

$$
\begin{aligned}
& \times E\left(\pi_{i}^{W}\left(b, b, \theta_{i}, \theta_{j}\right)-\pi_{i}^{L}\left(b, b, \theta_{i}, \theta_{j}\right) \mid \theta_{i}=\phi_{i}, \theta_{j} \leq \phi_{j}(b)\right) \\
& +E\left(\pi_{i}^{L}\left(b, b, \theta_{i}, \theta_{j}\right) \mid \theta_{i}=\phi_{i}, \theta_{j} \leq \phi_{j}(b)\right) \\
= & P\left(\theta_{j} \leq \phi_{j}(b) \mid \theta_{i}=\phi_{i}\right) E\left(\pi_{i}^{W}\left(b, b, \theta_{i}, \theta_{j}\right) \mid \theta_{i}=\phi_{i}, \theta_{j} \leq \phi_{j}(b)\right) \\
& +P\left(\theta_{j} \geq \phi_{j}(b) \mid \theta_{i}=\phi_{i}\right) E\left(\pi_{i}^{L}\left(b, b, \theta_{i}, \theta_{j}\right) \mid \theta_{i}=\phi_{i}, \theta_{j} \leq \phi_{j}(b)\right)
\end{aligned}
$$

[since $\pi_{i}^{L}$ is decreasing in $\theta_{j}$ by $\mathbf{A 1 1}$ ],

$$
\begin{aligned}
\geq & P\left(\theta_{j} \leq \phi_{j}(b) \mid \theta_{i}=\phi_{i}\right) E\left(\pi_{i}^{W}\left(b, b, \theta_{i}, \theta_{j}\right) \mid \theta_{i}=\phi_{i}, \theta_{j} \leq \phi_{j}(b)\right) \\
& +P\left(\theta_{j} \geq \phi_{j}(b) \mid \theta_{i}=\phi_{i}\right) E\left(\pi_{i}^{L}\left(b, b, \theta_{i}, \theta_{j}\right) \mid \theta_{i}=\phi_{i}, \theta_{j} \geq \phi_{j}(b)\right)
\end{aligned}
$$

[in view of A12]

$$
\begin{aligned}
\geq & P\left(\theta_{j} \leq \phi_{j}(b) \mid \theta_{i}=\phi_{i}\right) \\
& \times E\left(\pi_{i}^{W}\left(b, b_{j}\left(\theta_{j}\right), \theta_{i}, \theta_{j}\right) \mid \theta_{i}=\phi_{i}, \theta_{j} \leq \phi_{j}(b)\right) \\
& +P\left(\theta_{j} \geq \phi_{j}(b) \mid \theta_{i}=\phi_{i}\right) \\
& \times E\left(\pi_{i}^{L}\left(b, b_{j}\left(\theta_{j}\right), \theta_{i}, \theta_{j}\right) \mid \theta_{i}=\phi_{i}, \theta_{j} \geq \phi_{j}(b)\right) .
\end{aligned}
$$

Since the above expression is the payoff for a type $\phi_{i}$ bidding $b$, this is greater than the payoff for a type $\phi_{i}$ bidding just above $r$ (condition (QM)). This latter quantity is greater than 0 because $H_{i}^{r}\left(\psi_{i}, \psi_{j}\right)$ is quasimonotone in $\psi_{i}$.

In reference to our use of this lemma in our existence proof, notice that we have not made use of the equilibrium assumption in the proof of the lemma.

\section{REFERENCES}

Amman, E., and Leininger, W. (1996). "Asymmetric All-Pay Auctions with Incomplete Information: the Two-Player Case." Games Econom. Behavior 14, 1-18.

Athey, S. (1995). "Monotone Comparative Statics in Stochastic Optimization Problems," mimeo, MIT.

Athey, S. (1997). "Single Crossing Properties and the Existence of Pure Strategy Equilibria in Games of Incomplete Information," mimeo, MIT.

Bajari, P. (1996). "Properties of the First Price Sealed-Bid Auction with Asymmetric Bidders," mimeo, Stanford.

Bikchandani, S., and Riley, J. (1991). "Equilibrium in Open Common Value Auctions," J. Econom. Theory 53, 101-130. 
Dasgupta, P., and Maskin, E. (1986). "The Existence of Equilibrium in Discontinuous Games, I: Theory," Rev. Econom. Stud. 53, 1-26.

Fudenberg, D., and Tirole, J. (1986). "A Theory of Exit in Duopoly," Econometrica 54, 943960.

Hirsch, M., and Smale, S. (1974). Differential Equations, Dynamical Systems and Linear Algebra. Academic Press.

Jehiel, P., and Moldovanu, B. (1997). "Auctions with Downstream Interactions Among Buyers," mimeo, UCL.

Karamardian, S., and Schaible, S. (1990). "Seven Kinds of Monotone Maps,” J. Opt. Theory Appl. 66, 37-46.

Krishna, V., and Morgan, J. (1997). "An Analysis of the War of Attrition and the All-Pay Auction," J. Econom. Theory 72, 343-362.

Lebrun, B. (1994). "First Price Auction: Properties of the Equilibria in the Asymmetric N Bidders Case," mimeo.

Lebrun, B. (1996). "Existence of an Equilibrium in First Price Auctions," Econom. Theory 7, 421-445.

Maskin, E., and Riley, J. (1996). "Equilibrium in Sealed High Bid Auctions," mimeo, Harvard.

Maskin, E., and Riley, J. (1996). "Uniqueness in Sealed High Bid Auctions," mimeo, Harvard.

Milgrom, P. (1981). "Rational Expectations, Information Acquisition and Competitive Bidding," Econometrica 49, 921-943.

Milgrom, P., and Shannon, C. (1994). "Monotone Comparative Statics," Econometrica 62, 157180.

Milgrom, P., Weber, R. (1982). "A Theory of Auctions and Competitive Bidding,” Econometrica 50, 1089-1122.

Milgrom, P., and Weber, R. (1982). "Distributional Strategies for Games of Incomplete Information," Math. Oper. Res. 10, 619-632.

Nalebuff B., and Riley, J. (1985). "Asymmetric Equilibria in the War of Attrition,” J. Theor. Biology 113, 517-527.

Reny, P. (1997). "Local Payoff Security and the Existence of Pure and Mixed Strategy Nash Equilibria in Discontinuous Games," mimeo, Pittsburgh. 